\title{
PIECEWISE TENSOR PRODUCT WAVELET BASES BY EXTENSIONS AND APPROXIMATION RATES
}

\author{
NABI CHEGINI, STEPHAN DAHLKE, ULRICH FRIEDRICH, AND ROB STEVENSON
}

\begin{abstract}
Following [Studia Math., 76(2) (1983), pp. 1-58 and 95-136] by Z. Ciesielski and T. Figiel and [SIAM J. Math. Anal., 31 (1999), pp. 184-230] by W. Dahmen and R. Schneider, by the application of extension operators we construct a basis for a range of Sobolev spaces on a domain $\Omega$ from corresponding bases on subdomains that form a non-overlapping decomposition. As subdomains, we take hypercubes, or smooth parametric images of those, and equip them with tensor product wavelet bases. We prove approximation rates from the resulting piecewise tensor product basis that are independent of the spatial dimension of $\Omega$. For two- and three-dimensional polytopes we show that the solution of Poisson type problems satisfies the required regularity condition. The dimension independent rates will be realized numerically in linear complexity by the application of the adaptive wavelet-Galerkin scheme.
\end{abstract}

\section{INTRODUCTION}

Let $\Omega=\bigcup_{k=0}^{N} \Omega_{k} \subset \mathbb{R}^{n}$ be a non-overlapping domain decomposition. By the use of extension operators, we will construct isomorphisms from the Cartesian product of Sobolev spaces on the subdomains, which incorporate suitable boundary conditions, to Sobolev spaces on $\Omega$. By applying such an isomorphism to the union of Riesz bases for the Sobolev spaces on the subdomains, the result is a Riesz basis for the Sobolev space on $\Omega$.

Since the approach can be applied recursively, to understand the construction of such an isomorphism, it is sufficient to consider the case of having two subdomains. For $i \in\{1,2\}$, let $R_{i}$ be the restriction of functions on $\Omega$ to $\Omega_{i}$, let $\eta_{2}$ be the extension by zero of functions on $\Omega_{2}$ to functions on $\Omega$, and let $E_{1}$ be some extension of functions on $\Omega_{1}$ to functions on $\Omega$ which, for some $m \in \mathbb{N}_{0}$, is bounded from $H^{m}\left(\Omega_{1}\right)$ to the target space $H^{m}(\Omega)$. Then

$$
\left[\begin{array}{c}
R_{1} \\
R_{2}\left(\mathrm{Id}-E_{1} R_{1}\right)
\end{array}\right]: H^{m}(\Omega) \rightarrow H^{m}\left(\Omega_{1}\right) \times H_{0, \partial \Omega_{1} \cap \partial \Omega_{2}}^{m}\left(\Omega_{2}\right)
$$

Received by the editor September 2, 2011 and, in revised form, February 5, 2012 and February 14,2012 .

2010 Mathematics Subject Classification. Primary 15A69, 35B65, 41A25, 41A63, 42C40, $65 \mathrm{~N} 12,65 \mathrm{~T} 60$.

Key words and phrases. Wavelets, tensor product approximation, domain decomposition, extension operators, weighted anisotropic Sobolev space, regularity, adaptive wavelet scheme, best approximation rates, Fichera corner.

The first author was supported by the Netherlands Organization for Scientific Research (NWO) under contract no. 613.000.902.

The second and third authors were supported by Deutsche Forschungsgemeinschaft, grant number DA 360/12-1. The second author also acknowledges support by the LOEWE Center for Synthetic Microbiology, Marburg. 


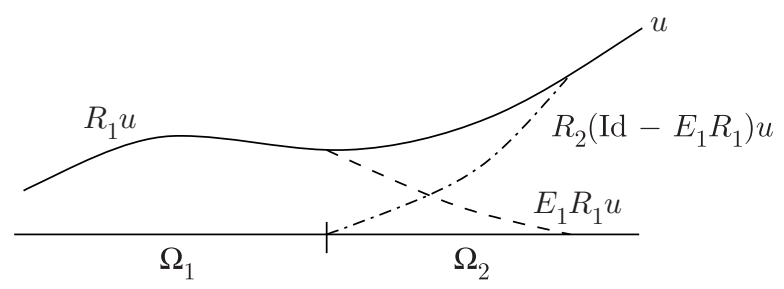

Figure 1. Splitting of $u$ into a sum of functions on the subdomains.
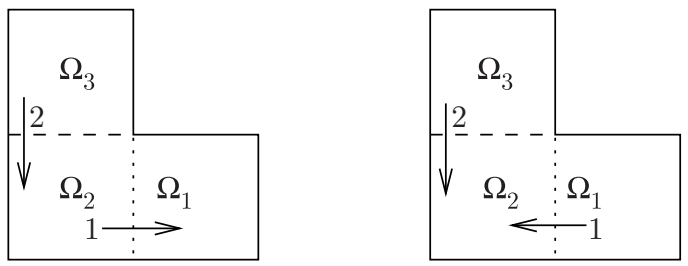

Figure 2. A feasible and a non-feasible configuration for $H^{1}(\Omega)$.

is boundedly invertible with inverse $\left[\begin{array}{ll}E_{1} & \eta_{2}\end{array}\right]$, see Figure $11\left(H_{0, \partial \Omega_{1} \cap \partial \Omega_{2}}^{m}\left(\Omega_{2}\right)\right.$ is the space of $H^{m}\left(\Omega_{2}\right)$ functions that vanish up to order $m-1$ at $\left.\partial \Omega_{1} \cap \partial \Omega_{2}\right)$. Consequently, if $\Psi_{1}$ is a Riesz basis for $H^{m}\left(\Omega_{1}\right)$ and $\Psi_{2}$ is a Riesz basis for $H_{0, \partial \Omega_{1} \cap \partial \Omega_{2}}^{m}\left(\Omega_{2}\right)$, then $E_{1} \Psi_{1} \cup \eta_{2} \Psi_{2}$ is a Riesz basis for $H^{m}(\Omega)$.

The principle to construct a basis for a function space on $\Omega$ by applying an isomorphism from this space onto the product of corresponding function spaces on non-overlapping subdomains was introduced in CF83. In DS99. (see also KS06]), this idea was revisited with the aim to practically construct such a basis for doing computations, rather than to show its existence.

In addition to the findings from DS99, in the current work we derive precise conditions on the ordering of the subdomains so that the corresponding "true" extension operators (not the trivial zero extensions), being the building blocks of the isomorphism, actually do exist as bounded mappings. To explain this, as an example, consider the construction of a basis for $H^{1}(\Omega)$ where $\Omega$ is an L-shaped domain subdivided into 3 subdomains as illustrated in Figure 2 The arrows depict the direction and the ordering of the extensions. The construction requires homogeneous boundary conditions on incoming interfaces and no boundary conditions on outgoing interfaces. In the left case we begin by constructing a basis for $H_{0, \partial \Omega_{2} \cap \partial \Omega_{3}}^{1}\left(\Omega_{1} \cup \Omega_{2}\right)$ as the union of a basis for $H_{0, \partial \Omega_{1} \cap \partial \Omega_{2}}^{1}\left(\Omega_{1}\right)$ and the image of a basis for $H_{0, \partial \Omega_{2} \cap \partial \Omega_{3}}^{1}\left(\Omega_{2}\right)$ under the first extension, which has to be bounded as an operator from $H_{0, \partial \Omega_{2} \cap \partial \Omega_{3}}^{1}\left(\Omega_{2}\right)$ to $H_{0, \partial \Omega_{2} \cap \partial \Omega_{3}}^{1}\left(\Omega_{1} \cup \Omega_{2}\right)$. The full basis is constructed by adding the image of a basis for $H^{1}\left(\Omega_{3}\right)$ under the second extension, which needs to be a bounded operator from $H^{1}\left(\Omega_{3}\right)$ to $H^{1}(\Omega)$.

Choosing the action of the extension operators as illustrated in the right case yields an invalid configuration. This is due to the fact that in the first step we would need a bounded extension operator from $H^{1}\left(\Omega_{1}\right)$ to $H_{0, \partial \Omega_{2} \cap \partial \Omega_{3}}^{1}\left(\Omega_{1} \cup \Omega_{2}\right)$. In 
view of the boundary condition incorporated in the latter space, this is, however, impossible.

The conditions on the directions of the arrows depend on the boundary conditions imposed on $\partial \Omega$, e.g., they will be different when a basis for $H_{0}^{1}(\Omega)$ is sought.

Our main interest in the construction of a basis from bases from subdomains lies in the use of piecewise tensor product approximation. On the hypercube

$$
\square:=(0,1)^{n},
$$

one can construct a basis for the Sobolev space $H^{m}(\square)$ (or for a subspace incorporating Dirichlet boundary conditions) by taking an $n$-fold tensor product of a collection of univariate functions that forms a Riesz basis for $L_{2}(0,1)$ as well as, properly scaled, for $H^{m}(0,1)$. Thinking of a univariate wavelet basis of order $d>m$, the advantage of this approach is that the rate of nonlinear best $M$-term approximation of a sufficiently smooth function $u$ is $d-m$, compared to $\frac{d-m}{n}$ for standard best $M$-term isotropic (wavelet) approximation of order $d$ on $\square$. The multiplication of the one-dimensional rate $d-m$ by the factor $\frac{1}{n}$ is commonly referred to as the curse of dimensionality.

One may argue that for any fixed $n$, a rate $d-m$ can also be obtained by isotropic approximation by increasing the order from $d$ to $n d-(n-1) m$. Concerning the required smoothness of $u$, however, in the latter case it is (essentially) necessary and sufficient that for $1 \leq i \leq n, 0 \leq k \leq m$, it holds that $\partial^{\alpha} \partial_{i}^{k} u \in L_{p}(\square)$ for $p=\left(d-m+\frac{1}{2}\right)^{-1}$ and $\|\alpha\|_{1} \leq n(d-m)$, where $\alpha$ denotes a multi-index, i.e., $\alpha \in \mathbb{N}_{0}^{n}$. With tensor product approximation the last condition reads as the much milder one $\|\alpha\|_{\infty} \leq d-m$ (a precise formulation of the smoothness conditions in terms of (tensor products of) Besov spaces can be found in [Nit06, SU09]).

Actually, the above conditions guarantee only any rate $s<d-m$. Arguments from interpolation space theory that are used do not give a result for the "endpoint" $s=d-m$.

In any case, for dimensions $n \geq 3$, the solution of an elliptic boundary value problem of order $2 m=2$ generally does not satisfy the conditions such that isotropic approximation converges with the best, or any near best possible rate allowed by the polynomial order, i.e., $\frac{d-m}{n}$ for order $d$. In order to achieve this rate, generally anisotropic approximation is mandatory (cf. Ape99).

In addition to avoiding the curse of dimensionality, the possibility of anisotropic approximation is automatically included in (adaptive) tensor product approximation. In DS10] (see also [Nit05]) it was shown that best approximations of $u$ from a suitably chosen nested sequence of spaces spanned by tensor product wavelets realize the best possible rate $d-m$, so not only any near best possible rate, when for $1 \leq i \leq n, 0 \leq k \leq m$ and $\|\alpha\|_{\infty} \leq d-m, \partial^{\alpha} \partial_{i}^{k} u$ is in a weighted $L_{2}(\square)$ space, with a weight being an $n$-fold product of univariate weights on $(0,1)$ that vanish at the endpoints. Clearly, the optimal rate $d-m$ for this linear approximation scheme implies this rate for the non-linear best $M$-term approximation from the tensor product basis. Furthermore, in DS10] it was shown that for a sufficiently smooth right-hand side, the solution of Poisson's problem on the $n$-dimensional unit cube $\square$ satisfies this regularity condition.

In view of these results on $\square$, we consider a domain $\Omega$ whose closure is the union of subdomains $\tau+\bar{\square}$ for some $\tau \in \mathbb{Z}^{n}$, or a domain $\Omega$ that is a parametric image of such a domain under a piecewise sufficiently smooth, globally $C^{m-1}$ diffeomorphism $\kappa$, being a homeomorphism when $m=1$. We equip $H^{m}(\Omega)$ (or 
a subspace incorporating Dirichlet boundary conditions) with a Riesz basis that is constructed using extension operators as discussed before from tensor product wavelet bases of order $d$ on the subdomains, or from push-forwards of such bases. Our restriction to decompositions of $\Omega$ into subdomains from a topological Cartesian partition will allow us to rely on univariate extensions. We will show the best possible approximation rate $d-m$ for any $u$ that restricted to any of these subdomains has a pull-back whose derivatives of sufficiently high order are in the aforementioned weighted $L_{2}(\square)$-spaces. The latter proof turns out to be technically difficult. Indeed, in order to end up with locally supported wavelets, we will apply local, scale-dependent extension operators, i.e., only wavelets that are non-zero near an interface will be extended, which do not preserve more smoothness than essentially membership of $H^{m}$.

Furthermore, using anisotropic regularity results recently shown in [CDN10], we show that if, additionally, $\Omega$ is a two- or, more interesting, a three-dimensional polytope, then for a sufficiently smooth right-hand side, the solution of Poisson's problem satisfies this piecewise regularity condition. For that to hold in three dimensions, it will be needed that the parametrization map $\kappa$ is piecewise trilinear, and it may require a refinement of the initial decomposition of $\Omega$.

Since it defines a boundedly invertible mapping from a Hilbert space, being $H_{0}^{1}(\Omega)$, to its dual, the Poisson problem is an example of a well-posed operator equation. Equipping $H_{0}^{1}(\Omega)$ with a Riesz basis constructed using extension operators from tensor product wavelet bases of order $d$ on the subdomains, the operator equation is equivalently formulated as a boundedly invertible bi-infinite matrix vector equation. Approximate solutions produced by the adaptive wavelet-Galerkin method (CDD01, Ste09]) were proven to converge with the best possible rate in linear complexity. We perform numerical tests in two and three dimensions with wavelets of order $d=5$ that confirm that this rate is $d-m$.

This paper is organized as follows: In Section 2, we present the abstract idea behind the construction of isomorphisms from a Sobolev space on a domain onto the product of corresponding Sobolev spaces on subdomains that form a nonoverlapping decomposition.

In Section 3, we recall results on tensor product approximation on a hypercube, and collect assumptions on the univariate wavelets, being the building blocks of the tensor product wavelets.

In Section 4 we consider a domain $\Omega$ that is the union of hypercubes from a Cartesian partition of $\mathbb{R}^{n}$ into hypercubes. We formulate precise conditions on the order in which univariate extensions over interfaces have to be applied, and which boundary conditions have to be imposed, such that for a range of smoothness indices the composition of these extensions is an isomorphism from a Sobolev spaces on $\Omega$ onto the product of the corresponding Sobolev spaces on the collection of hypercubes. Equipping these hypercubes with tensor product wavelet bases, we end up with a piecewise tensor product wavelet basis on $\Omega$.

In order to obtain locally supported primal and dual wavelets, in Section 5 the extension operators are replaced by scale-dependent modifications, in the sense that only wavelets with supports "near" the interfaces are extended. It is shown that approximation from the resulting piecewise tensor product basis gives rise to rates that are independent of the spatial dimension, assuming the function that is approximated satisfies some mild, piecewise weighted Sobolev smoothness conditions. 
In Section 6, these regularity conditions are verified for the solution of Poisson's problem with sufficiently smooth right-hand side in two and three-dimensional polytopes.

The best possible rates from the piecewise tensor product basis can be realized in linear complexity by the application of the adaptive wavelet-Galerkin scheme. In Section 7, we present numerical results obtained with this scheme for the twodimensional slit domain, and the three-dimensional thick L-shaped domain and the Fichera corner domain.

\section{Construction of the isomorphisms}

In an abstract setting, for a class of mappings from a Banach space to the Cartesian product of two other Banach spaces, we give conditions on such mappings to be isomorphisms. The results will be applied to construct isomorphisms from a Sobolev space on a domain onto the product of Sobolev spaces on subdomains.

Proposition 2.1. For normed linear spaces $V$ and $V_{i}(i=1,2)$, let $E_{1} \in B\left(V_{1}, V\right)$, $\eta_{2} \in B\left(V_{2}, V\right), R_{1} \in B\left(V, V_{1}\right)$, and $R_{2} \in B\left(\Im \eta_{2}, V_{2}\right)$ be such that

$$
R_{1} E_{1}=\mathrm{Id}, \quad R_{2} \eta_{2}=\mathrm{Id}, \quad R_{1} \eta_{2}=0, \quad \Im\left(\mathrm{Id}-E_{1} R_{1}\right) \subset \Im \eta_{2} .
$$

Then

$$
E=\left[\begin{array}{ll}
E_{1} & \eta_{2}
\end{array}\right] \in B\left(V_{1} \times V_{2}, V\right) \text { is boundedly invertible, }
$$

with inverse

$$
E^{-1}=\left[\begin{array}{c}
R_{1} \\
R_{2}\left(\mathrm{Id}-E_{1} R_{1}\right)
\end{array}\right] .
$$

Proof. Using that $R_{1} E_{1}=\mathrm{Id}, R_{1} \eta_{2}=0, R_{2} \eta_{2}=\mathrm{Id}$, we have

$$
\left[\begin{array}{c}
R_{1} \\
R_{2}\left(\mathrm{Id}-E_{1} R_{1}\right)
\end{array}\right]\left[\begin{array}{ll}
E_{1} & \eta_{2}
\end{array}\right]=\left[\begin{array}{cc}
\mathrm{Id} & 0 \\
0 & \mathrm{Id}
\end{array}\right],
$$

and using that $\Im\left(\mathrm{Id}-E_{1} R_{1}\right) \subset \Im \eta_{2}$ and $R_{2} \eta_{2}=\mathrm{Id}$, we have

$$
\left[\begin{array}{ll}
E_{1} & \eta_{2}
\end{array}\right]\left[\begin{array}{c}
R_{1} \\
R_{2}\left(\mathrm{Id}-E_{1} R_{1}\right)
\end{array}\right]=E_{1} R_{1}+\eta_{2} R_{2}\left(\mathrm{Id}-E_{1} R_{1}\right)=\mathrm{Id}
$$

In applications $V\left(V_{i}\right)$ will be densely embedded in a Hilbert space $H\left(H_{i}\right)$. Questions about boundedness of $E$ or $E^{-1}$ in dual spaces then reduce to properties of the Hilbert adjoint of $E$. Study of the Hilbert adjoint will also be relevant for the investigation of dual bases.

Proposition 2.2. For Hilbert spaces $H$ and $H_{i}(i=1,2)$, let $R_{i} \in B\left(H, H_{i}\right)$, and isometries $\eta_{i} \in B\left(H_{i}, H\right)$ be such that

$$
R_{i} \eta_{j}=\delta_{i j} \quad(i, j \in\{1,2\}), \quad H=\Im \eta_{1} \oplus^{\perp} \Im \eta_{2},
$$

and let $E_{1} \in B\left(H_{1}, H\right)$ be such that $R_{1} E_{1}=\mathrm{Id}$.

Then $\eta_{1} R_{1}+\eta_{2} R_{2}=\mathrm{Id}, E \in B\left(H_{1} \times H_{2}, H\right)$ is boundedly invertible, $\eta_{i}^{*}=R_{i}$, and

$$
E^{*}=\left[\begin{array}{c}
E_{1}^{*} \\
R_{2}
\end{array}\right], \quad E^{-*}=\left[\eta_{1} \quad\left(\mathrm{Id}-\eta_{1} E_{1}^{*}\right) \eta_{2}\right] .
$$


Proof. The first statement follows from $\eta_{1} R_{1}+\eta_{2} R_{2}=$ Id on $\Im \eta_{i}$. The second statement follows from Proposition 2.1 once we have verified that $\Im\left(\operatorname{Id}-E_{1} R_{1}\right) \subset$ $\Im \eta_{2}$. Writing $\left(\mathrm{Id}-E_{1} R_{1}\right) x=\eta_{1} x_{1}+\eta_{2} x_{2}$, and applying $R_{1}$ to both sides, we find $x_{1}=0$ as required. For any $u \in H_{i}, v \in H$,

$$
\left\langle\eta_{i} u, v\right\rangle_{H}=\left\langle\eta_{i} u, \sum_{j} \eta_{j} R_{j} v\right\rangle_{H}=\left\langle\eta_{i} u, \eta_{i} R_{i} v\right\rangle_{H}=\left\langle u, R_{i} v\right\rangle_{H_{i}},
$$

or $\eta_{i}^{*}=R_{i}$. Now the last statement follows from the formulas for $E$ and $E^{-1}$ given in Proposition 2.1]

Remark 2.3. The formulas for $E$ and $E^{-*}$, and so those for $E^{-1}$ and $E^{*}$ are symmetric, with reversed roles of $H_{1}$ and $H_{2}$, in the sense that with $E_{2}:=\left(\operatorname{Id}-\eta_{1} E_{1}^{*}\right) \eta_{2}$, it holds that $\left(\mathrm{Id}-\eta_{2} E_{2}^{*}\right) \eta_{1}=E_{1}$.

Let $\tilde{V}$ and $\tilde{V}_{i}(i=1,2)$ be reflexive Banach spaces with

$$
\tilde{V} \hookrightarrow H, \quad \tilde{V}_{i} \hookrightarrow H_{i} \text { with dense embeddings. }
$$

In this setting, we have that boundedness, or bounded invertibility of

$$
E: \tilde{V}_{1}^{\prime} \times \tilde{V}_{2}^{\prime} \rightarrow \tilde{V}^{\prime}
$$

is equivalent to boundedness, or to bounded invertibility of

$$
E^{*}: \tilde{V} \rightarrow \tilde{V}_{1} \times \tilde{V}_{2} .
$$

Proposition 2.4. Let the assumptions of Proposition 2.2 be valid. Let

$$
R_{2} \in B\left(\tilde{V}, \tilde{V}_{2}\right), \quad \eta_{1} \in B\left(\tilde{V}_{1}, \tilde{V}\right), \quad E_{1}^{*} \in B\left(\tilde{V}, \tilde{V}_{1}\right) .
$$

Then $E^{*} \in B\left(\tilde{V}, \tilde{V}_{1} \times \tilde{V}_{2}\right)$, and so $E \in B\left(\tilde{V}_{1}^{\prime} \times \tilde{V}_{2}^{\prime}, \tilde{V}^{\prime}\right)$, is boundedly invertible if and only if $R_{2}$ has a right-inverse $\hat{E}_{2} \in B\left(\tilde{V}_{2}, \tilde{V}\right)$.

Proof. The assumptions imply that $E^{*} \in B\left(\tilde{V}, \tilde{V}_{1} \times \tilde{V}_{2}\right)$, and that for $E^{-*} \in B\left(\tilde{V}_{1} \times\right.$ $\left.\tilde{V}_{2}, \tilde{V}\right)$ it suffices to show that $E_{2}:=\left(\operatorname{Id}-\eta_{1} E_{1}^{*}\right) \eta_{2} \in B\left(\tilde{V}_{2}, \tilde{V}\right)$. If the latter is true, then, since $R_{2} E_{2}=\mathrm{Id}$, we can take $\hat{E}_{2}=E_{2}$.

Conversely, let $\hat{E}_{2} \in B\left(\tilde{V}_{2}, \tilde{V}\right)$ be a right-inverse of $R_{2}$. We have that

$$
\begin{aligned}
R_{1}\left(\mathrm{Id}-E_{2} R_{2}\right) & =R_{1}-R_{1} \eta_{2} R_{2}+R_{1} \eta_{1} E_{1}^{*} \eta_{2} R_{2}=R_{1}+E_{1}^{*} \eta_{2} R_{2} \\
& =E_{1}^{*}\left(\eta_{1} R_{1}+\eta_{2} R_{2}\right)=E_{1}^{*} \in B\left(\tilde{V}, \tilde{V}_{1}\right) .
\end{aligned}
$$

So

$$
\mathrm{Id}-E_{2} R_{2}=\left(\eta_{1} R_{1}+\eta_{2} R_{2}\right)\left(\mathrm{Id}-E_{2} R_{2}\right)=\eta_{1} R_{1}\left(\mathrm{Id}-E_{2} R_{2}\right) \in B(\tilde{V}, \tilde{V}),
$$

or $E_{2} R_{2} \in B(\tilde{V}, \tilde{V})$. But then $E_{2}=E_{2} R_{2} \hat{E}_{2} \in B\left(\tilde{V}_{2}, \tilde{V}\right)$.

Finally in this section, we apply arguments from interpolation space theory to conclude boundedness of $E$ in scales of Banach spaces.

Proposition 2.5. (a) Let $V, \underline{V}$, and $V_{i}, \underline{V}_{i}(i=1,2)$ be Banach spaces with

$$
\underline{V} \hookrightarrow V, \quad \underline{V}_{i} \hookrightarrow V_{i} \quad \text { with dense embeddings. }
$$

Let the mappings $\left(R_{1}, R_{2}, E_{1}, \eta_{2}\right)$ satisfy the conditions from Proposition 2.1 for both triples $\left(V, V_{1}, V_{2}\right)$ and $\left(\underline{V}, \underline{V}_{1}, \underline{V}_{2}\right)$. Then for $s \in[0,1], q \in[1, \infty]$,

$$
E \in B\left(\left[V_{1}, \underline{V}_{1}\right]_{s, q} \times\left[V_{2}, \underline{V}_{2}\right]_{s, q},[V, \underline{V}]_{s, q}\right) \text { is boundedly invertible. }
$$


(b) Let $\tilde{V}, \underline{\tilde{V}}$, and $\tilde{V}_{i}, \tilde{V}_{i}$ be reflexive Banach spaces, and $H$ and $H_{i}$ be Hilbert spaces $(i=1,2)$ with

$$
\underline{\tilde{V}} \hookrightarrow \tilde{V} \hookrightarrow H, \quad \underline{\tilde{V}}_{i} \hookrightarrow \tilde{V}_{i} \hookrightarrow H_{i} \quad \text { with dense embeddings. }
$$

Let the conditions of Proposition 2.2 be satisfied, as well as the conditions of Proposition 2.4 for both triples $\left(\tilde{V}, \tilde{V}_{1}, \tilde{V}_{2}\right)$ and $\left(\underline{\tilde{V}}, \underline{\tilde{V}}_{1}, \tilde{V}_{2}\right)$. Then for $s \in[0,1]$, $q \in[1, \infty]$,

$$
E \in B\left(\left[\tilde{V}_{1}, \tilde{\tilde{V}}_{1}\right]_{s, q}^{\prime} \times\left[\tilde{V}_{2}, \underline{\tilde{V}}_{2}\right]_{s, q}^{\prime},[\tilde{V}, \underline{\tilde{V}}]_{s, q}^{\prime}\right) \text { is boundedly invertible. }
$$

\section{Approximation by tensor product WaVElets on the hypercube}

We will study non-overlapping domain decompositions, where the subdomains are either unit $n$-cubes or smooth images of those. Sobolev spaces on these cubes, that appear with the construction of a Riesz basis for a Sobolev space on the domain as a whole, will be equipped with tensor product wavelet bases. From [DS10, we recall the construction of those bases, as well as results on the rate of approximation from spans of suitably chosen subsets of these bases. let

For $t \in[0, \infty) \backslash\left(\mathbb{N}_{0}+\left\{\frac{1}{2}\right\}\right)$ and $\vec{\sigma}=\left(\sigma_{\ell}, \sigma_{r}\right) \in\left\{0, \ldots,\left\lfloor t+\frac{1}{2}\right\rfloor\right\}^{2}$, with $\mathcal{I}:=(0,1)$,

$$
H_{\vec{\sigma}}^{t}(\mathcal{I}):=\left\{v \in H^{t}(\mathcal{I}): v(0)=\cdots=v^{\left(\sigma_{\ell}-1\right)}(0)=0=v(1)=\cdots=v^{\left(\sigma_{r}-1\right)}(1)\right\} .
$$

Remark 3.1. Later, we will use this definition also with $\mathcal{I}$ reading as a general non-empty interval, with 0 and 1 reading as its left and right boundary.

For $t$ and $\vec{\sigma}$ as above, and for $\tilde{t} \in[0, \infty) \backslash\left(\mathbb{N}_{0}+\left\{\frac{1}{2}\right\}\right)$ and $\overrightarrow{\tilde{\sigma}}=\left(\tilde{\sigma}_{\ell}, \tilde{\sigma}_{r}\right) \in$ $\left\{0, \ldots,\left\lfloor\tilde{t}+\frac{1}{2}\right\rfloor\right\}^{2}$, we assume univariate wavelet collections

$$
\Psi_{\vec{\sigma}, \overrightarrow{\tilde{\sigma}}}:=\left\{\psi_{\lambda}^{(\vec{\sigma}, \overrightarrow{\tilde{\sigma}})}: \lambda \in \nabla_{\vec{\sigma}, \overrightarrow{\tilde{\sigma}}}\right\} \subset H_{\vec{\sigma}}^{t}(\mathcal{I})
$$

such that

$\left(\mathcal{W}_{1}\right) \Psi_{\vec{\sigma}, \overrightarrow{\tilde{\sigma}}}$ is a Riesz basis for $L_{2}(\mathcal{I})$,

$\left(\mathcal{W}_{2}\right)\left\{2^{-|\lambda| t} \psi_{\lambda}^{(\vec{\sigma}, \overrightarrow{\tilde{\sigma}})}: \lambda \in \nabla_{\vec{\sigma}, \vec{\sigma}}\right\}$ is a Riesz basis for $H_{\vec{\sigma}}^{t}(\mathcal{I})$,

where $|\lambda| \in \mathbb{N}_{0}$ denotes the level of $\lambda$. Denoting the dual basis of $\Psi_{\vec{\sigma}, \vec{\sigma}}$ for $L_{2}(\mathcal{I})$ as $\tilde{\Psi}_{\vec{\sigma}, \overrightarrow{\tilde{\sigma}}}:=\left\{\tilde{\psi}_{\lambda}^{(\vec{\sigma}, \overrightarrow{\tilde{\sigma}})}: \lambda \in \nabla_{\vec{\sigma}, \overrightarrow{\tilde{\sigma}}}\right\}$, furthermore we assume that

$\left(\mathcal{W}_{3}\right)\left\{2^{-|\lambda| \tilde{t}} \tilde{\psi}_{\lambda}^{(\vec{\sigma}, \overrightarrow{\tilde{\sigma}})}: \lambda \in \nabla_{\vec{\sigma}, \overrightarrow{\tilde{\sigma}}}\right\}$ is a Riesz basis for $H_{\overrightarrow{\tilde{\sigma}}}^{\tilde{t}}(\mathcal{I})$, and that for some

$$
\mathbb{N} \ni d>t
$$

$$
\begin{aligned}
& \left(\mathcal{W}_{4}\right)\left|\left\langle\tilde{\psi}_{\lambda}^{(\vec{\sigma}, \overrightarrow{\tilde{\sigma}})}, u\right\rangle_{L_{2}(\mathcal{I})}\right| \lesssim 2^{-|\lambda| d}\|u\|_{H^{d}(\operatorname{supp} \tilde{\psi}(\vec{\sigma}, \vec{\sigma})}\left(u \in H^{d}(\mathcal{I}) \cap H_{\vec{\sigma}}^{t}(\mathcal{I})\right), \\
& \left(\mathcal{W}_{5}\right) \quad \rho:=\sup _{\lambda \in \nabla_{\vec{\sigma}, \vec{\sigma}}} 2^{|\lambda|} \max \left(\operatorname{diam} \operatorname{supp} \tilde{\psi}_{\lambda}^{(\vec{\sigma}, \vec{\sigma})}, \operatorname{diam} \operatorname{supp} \psi_{\lambda}^{(\vec{\sigma}, \vec{\sigma})}\right) \\
& \approx \inf _{\lambda \in \nabla_{\vec{\sigma}, \vec{\sigma}}} 2^{|\lambda|} \max \left(\operatorname{diam} \operatorname{supp} \tilde{\psi}_{\lambda}^{(\vec{\sigma}, \overrightarrow{\tilde{\sigma}})}, \operatorname{diam} \operatorname{supp} \psi_{\lambda}^{(\vec{\sigma}, \vec{\sigma})}\right), \\
& \left(\mathcal{W}_{6}\right) \sup _{j, k \in \mathbb{N}_{0}} \#\left\{|\lambda|=j:\left[k 2^{-j},(k+1) 2^{-j}\right] \cap\left(\operatorname{supp} \tilde{\psi}_{\lambda}^{(\vec{\sigma}, \overrightarrow{\tilde{\sigma}})} \cup \operatorname{supp} \psi_{\lambda}^{(\vec{\sigma}, \overrightarrow{\tilde{\sigma}})}\right) \neq \emptyset\right\}<\infty .
\end{aligned}
$$

The conditions $\left(\mathcal{W}_{5}\right)$ and $\left(\mathcal{W}_{6}\right)$ will be referred to by saying that both primal and dual wavelets are local or locally finite, respectively. For some arguments, it will be used that by increasing the coarsest scale, the constant $\rho$ can always be assumed to be sufficiently small. 
With, for $n \in \mathbb{N}$,

$$
\square:=\mathcal{I}^{n},
$$

one has $L_{2}(\square)=\bigotimes_{i=1}^{n} L_{2}(\mathcal{I})$. For

$$
\boldsymbol{\sigma}=\left(\vec{\sigma}_{i}=\left(\left(\sigma_{i}\right)_{\ell},\left(\sigma_{i}\right)_{r}\right)\right)_{1 \leq i \leq n} \in\left(\left\{0, \ldots,\left\lfloor t+\frac{1}{2}\right\rfloor\right\}^{2}\right)^{n},
$$

we define

$$
H_{\boldsymbol{\sigma}}^{t}(\square):=H_{\vec{\sigma}_{1}}^{t}(\mathcal{I}) \otimes L_{2}(\mathcal{I}) \otimes \cdots \otimes L_{2}(\mathcal{I}) \cap \cdots \cap L_{2}(\mathcal{I}) \otimes \cdots \otimes L_{2}(\mathcal{I}) \otimes H_{\vec{\sigma}_{n}}^{t}(\mathcal{I}),
$$

which is the space of $H^{t}(\square)$-functions whose normal derivatives of up to orders $\left(\sigma_{i}\right)_{\ell}$ and $\left(\sigma_{i}\right)_{r}$ vanish at the facets $\overline{\mathcal{I}}^{i-1} \times\{0\} \times \overline{\mathcal{I}}^{n-i}$ and $\overline{\mathcal{I}}^{i-1} \times\{1\} \times \overline{\mathcal{I}}^{n-i}$, respectively, $(1 \leq i \leq n)$ (the proof of this fact given in DS10 for $t \in \mathbb{N}_{0}$ can be generalized to $\left.t \in[0, \infty) \backslash\left(\mathbb{N}_{0}+\frac{1}{2}\right)\right)$.

The tensor product wavelet collection

$$
\Psi_{\boldsymbol{\sigma}, \tilde{\boldsymbol{\sigma}}}:=\bigotimes_{i=1}^{n} \Psi_{\vec{\sigma}_{i}, \vec{\sigma}_{i}}=\left\{\boldsymbol{\psi}_{\boldsymbol{\lambda}}^{(\boldsymbol{\sigma}, \tilde{\boldsymbol{\sigma}})}:=\bigotimes_{i=1}^{n} \psi_{\lambda_{i}}^{\left(\vec{\sigma}_{i}, \overrightarrow{\tilde{\sigma}}_{i}\right)}: \boldsymbol{\lambda} \in \nabla_{\boldsymbol{\sigma}, \tilde{\boldsymbol{\sigma}}}:=\prod_{i=1}^{n} \nabla_{\vec{\sigma}_{i}, \vec{\sigma}_{i}}\right\},
$$

and its renormalized version $\left\{\left(\sum_{i=1}^{n} 4^{t\left|\lambda_{i}\right|}\right)^{-1 / 2} \boldsymbol{\psi}_{\boldsymbol{\lambda}}^{(\boldsymbol{\sigma}, \tilde{\boldsymbol{\sigma}})}: \boldsymbol{\lambda} \in \boldsymbol{\nabla}_{\boldsymbol{\sigma}, \tilde{\boldsymbol{\sigma}}}\right\}$ are Riesz bases for $L_{2}(\square)$ and $H_{\boldsymbol{\sigma}}^{t}(\square)$, respectively. The collection that is dual to $\boldsymbol{\Psi}_{\boldsymbol{\sigma}, \tilde{\boldsymbol{\sigma}}}$ reads as

$$
\tilde{\boldsymbol{\Psi}}_{\boldsymbol{\sigma}, \tilde{\boldsymbol{\sigma}}}:=\bigotimes_{i=1}^{n} \tilde{\Psi}_{\vec{\sigma}_{i}, \overrightarrow{\tilde{\sigma}}_{i}}=\left\{\tilde{\boldsymbol{\psi}}_{\boldsymbol{\lambda}}^{(\boldsymbol{\sigma}, \tilde{\boldsymbol{\sigma}})}:=\bigotimes_{i=1}^{n} \tilde{\psi}_{\lambda_{i}}^{\left(\vec{\sigma}_{i}, \overrightarrow{\tilde{\sigma}}_{i}\right)}: \boldsymbol{\lambda} \in \nabla_{\boldsymbol{\sigma}, \tilde{\boldsymbol{\sigma}}\}},\right.
$$

and its renormalized version $\left\{\left(\sum_{i=1}^{n} 4^{\left|\lambda_{i}\right|}\right)^{-\tilde{t} / 2} \tilde{\boldsymbol{\psi}}_{\boldsymbol{\lambda}}^{(\boldsymbol{\sigma}, \tilde{\boldsymbol{\sigma}})}: \boldsymbol{\lambda} \in \boldsymbol{\nabla}_{\boldsymbol{\sigma}, \tilde{\boldsymbol{\sigma}}}\right\}$ is a Riesz basis for $H_{\tilde{\sigma}}^{\tilde{t}}(\square)$.

For $\boldsymbol{\lambda} \in \nabla_{\boldsymbol{\sigma}, \tilde{\boldsymbol{\sigma}}}$, we set $|\boldsymbol{\lambda}|:=\left(\left|\lambda_{1}\right|, \ldots,\left|\lambda_{n}\right|\right)$. As usual, for $\boldsymbol{j}, \boldsymbol{\jmath} \in \mathbb{N}_{0}^{n},|\boldsymbol{j}| \leq|\boldsymbol{\jmath}|$ will mean that $|\boldsymbol{j}|_{i} \leq|\boldsymbol{J}|_{i}(1 \leq i \leq n)$, whereas $|\boldsymbol{j}| \geq|\boldsymbol{\jmath}|$ or $|\boldsymbol{j}|=|\boldsymbol{\jmath}|$ will mean that $|\boldsymbol{J}| \leq|\boldsymbol{j}|$ or $|\boldsymbol{j}| \leq|\boldsymbol{\jmath}|$ and $|\boldsymbol{j}| \geq|\boldsymbol{\jmath}|$, respectively.

For $\theta \geq 0$, the weighted Sobolev space $\mathcal{H}_{\theta}^{d}(\mathcal{I})$ is defined as the space of all measurable functions $u$ on $\mathcal{I}$ for which the norm

$$
\|u\|_{\mathcal{H}_{\theta}^{d}(\mathcal{I})}:=\left[\sum_{j=0}^{d} \int_{\mathcal{I}}\left|x^{\theta}(1-x)^{\theta} u^{(j)}(x)\right|^{2} d x\right]^{\frac{1}{2}}
$$

is finite. For

$$
m \in\{0, \ldots,\lfloor t\rfloor\},
$$

we will consider the weighted Sobolev space

$$
\mathcal{H}_{m, \theta}^{d}(\square):=\bigcap_{p=1}^{n} \bigotimes_{i=1}^{n} \mathcal{H}_{\theta-\delta_{i p} \min (m, \theta)}^{d}(\mathcal{I})
$$

equipped with a squared norm that is the sum over $p=1, \ldots, n$ of the squared norms on $\bigotimes_{i=1}^{n} \mathcal{H}_{\theta-\delta_{i p} \min (m, \theta)}^{d}(\mathcal{I})$.

Theorem 3.2 ([DS10, Thm. 4.3]). For any $\theta \in[0, d)$, there exist a (nested) sequence $\left(\boldsymbol{\nabla}_{M}^{(\boldsymbol{\sigma}, \tilde{\boldsymbol{\sigma}})}\right)_{M \in \mathbb{N}} \subset \boldsymbol{\nabla}_{\boldsymbol{\sigma}, \tilde{\boldsymbol{\sigma}}}$ with $\# \boldsymbol{\nabla}_{M}^{(\boldsymbol{\sigma}, \tilde{\boldsymbol{\sigma}})} \bar{\sim}$, such that

$$
\begin{aligned}
& \inf _{v \in \operatorname{span}\left\{\psi_{\lambda}^{(\sigma, \tilde{\sigma})}: \boldsymbol{\lambda} \in \nabla_{M}^{(\sigma, \tilde{\sigma})}\right\}}\|u-v\|_{H^{m}(\square)} \\
& \lesssim M^{-(d-m)}\|u\|_{\mathcal{H}_{m, \theta}^{d}(\square)}, \quad\left(u \in \mathcal{H}_{m, \theta}^{d}(\square) \cap H_{\sigma}^{m}(\square)\right)
\end{aligned}
$$


where for $m=0, M^{-(d-m)}$ should be read as $(\log \# M)^{(n-1)\left(\frac{1}{2}+d\right)} M^{-d}$.

The index sets $\boldsymbol{\nabla}_{M}^{(\boldsymbol{\sigma}, \tilde{\boldsymbol{\sigma}})}$ can be chosen to have the following multiple tree property: For any $\boldsymbol{\lambda} \in \boldsymbol{\nabla}_{M}^{(\boldsymbol{\sigma}, \tilde{\boldsymbol{\sigma}})}$ and any $\boldsymbol{j} \in \mathbb{N}_{0}^{n}$ with $\boldsymbol{j} \leq|\boldsymbol{\lambda}|$, there exists $a \boldsymbol{\mu} \in \boldsymbol{\nabla}_{M}^{(\boldsymbol{\sigma}, \tilde{\boldsymbol{\sigma}})}$ with $|\boldsymbol{\mu}|=\boldsymbol{j}$, and $\operatorname{supp} \boldsymbol{\psi}_{\boldsymbol{\lambda}}^{(\boldsymbol{\sigma}, \tilde{\boldsymbol{\sigma}})} \cap \operatorname{supp} \boldsymbol{\psi}_{\boldsymbol{\mu}}^{(\boldsymbol{\sigma}, \tilde{\boldsymbol{\sigma}})} \neq \emptyset$.

With the notations $u \in H_{\boldsymbol{\sigma}}^{t}(\alpha+\square)$ and $u \in \mathcal{H}_{m, \theta}^{d}(\alpha+\square)$, we will mean that $u(\cdot+\alpha) \in H_{\boldsymbol{\sigma}}^{t}(\square)$ or $u(\cdot+\alpha) \in \mathcal{H}_{m, \theta}^{d}(\square)$, respectively.

\section{Construction of Riesz bases by extension}

Let $\left\{\square_{0}, \ldots, \square_{N}\right\}$ be a set of hypercubes from $\left\{\tau+\square: \tau \in \mathbb{Z}^{n}\right\}$, and let $\hat{\Omega}$ be a (reference) domain (i.e., open and connected) in $\mathbb{R}^{n}$ with $\bigcup_{k=0}^{N} \square_{k} \subset \hat{\Omega} \subset$ $\left(\bigcup_{k=0}^{N} \bar{\square}_{k}\right)^{\text {int }}$, and such that $\partial \hat{\Omega}$ is the union of (closed) facets of the $\square_{k}$ 's. The case $\hat{\Omega} \subsetneq\left(\bigcup_{k=0}^{N} \bar{\square}_{k}\right)^{\text {int }}$ corresponds to the situation that $\hat{\Omega}$ has one or more cracks. We will describe a construction of Riesz bases for Sobolev spaces on $\hat{\Omega}$ from Riesz bases for corresponding Sobolev spaces on the subdomains $\square_{k}$ using extension operators. We start by giving sufficient conditions $\left(\mathcal{D}_{1}-\mathcal{D}_{5}\right.$ such that suitable extension operators exist. At the end of this section, we will consider domains given as the parametric image of $\hat{\Omega}$.

We assume that there exists a sequence $\left(\left\{\hat{\Omega}_{k}^{(q)}: q \leq k \leq N\right\}\right)_{0 \leq q \leq N}$ of sets of polytopes, such that $\hat{\Omega}_{k}^{(0)}=\square_{k}$ and where each next term in the sequence is created from its predecessor by joining two of its polytopes. More precisely, we assume that for any $1 \leq q \leq N$, there exists a $q \leq \bar{k}=\bar{k}^{(q)} \leq N$ and $q-1 \leq k_{1}=k_{1}^{(q)} \neq k_{2}=$ $k_{2}^{(q)} \leq N$ such that

$\left(\mathcal{D}_{1}\right) \hat{\Omega}_{\bar{k}}^{(q)}=\left(\overline{\hat{\Omega}_{k_{1}}^{(q-1)} \cup \hat{\Omega}_{k_{2}}^{(q-1)}} \backslash \partial \hat{\Omega}\right)^{\text {int }}$ is connected, and the interface $J:=\hat{\Omega}_{\bar{k}}^{(q)} \backslash$ $\left(\hat{\Omega}_{k_{1}}^{(q-1)} \cup \hat{\Omega}_{k_{2}}^{(q-1)}\right)$ is part of a hyperplane,

$\left(\mathcal{D}_{2}\right)\left\{\hat{\Omega}_{k}^{(q)}: q \leq k \leq N, k \neq \bar{k}\right\}=\left\{\hat{\Omega}_{k}^{(q-1)}: q-1 \leq k \leq N, k \neq\left\{k_{1}, k_{2}\right\}\right\}$,

$\left(\mathcal{D}_{3}\right) \hat{\Omega}_{N}^{(N)}=\hat{\Omega}$.

For some

$$
t \in[0, \infty) \backslash\left(\mathbb{N}_{0}+\left\{\frac{1}{2}\right\}\right),
$$

to each of the closed facets of all the hypercubes $\square_{k}$, we associate a number in $\left\{0, \ldots,\left\lfloor t+\frac{1}{2}\right\rfloor\right\}$ indicating the order of the Dirichlet boundary condition on that facet (where a Dirichlet boundary condition of order 0 means no boundary condition). On facets on the boundary of $\hat{\Omega}$, this number can be chosen at one's convenience (it is dictated by the boundary conditions of the boundary value problem that one wants to solve on $\hat{\Omega}$ ), and, as will follow from the conditions imposed below, on the other facets it should be either 0 or $\left\lfloor t+\frac{1}{2}\right\rfloor$.

By construction, each facet of any $\hat{\Omega}_{k}^{(q)}$ is a union of some facets of the $\square_{k}$ 's, that will be referred to as subfacets. Letting each of these subfacets inherit the Dirichlet boundary conditions imposed on the $\square_{k^{\prime}}$ 's, we define

$$
\stackrel{\circ}{H}^{t}\left(\hat{\Omega}_{k}^{(q)}\right)
$$

and so for $k=q=N$, in particular $\stackrel{\circ}{H}^{t}(\hat{\Omega})=\stackrel{\circ}{H}^{t}\left(\hat{\Omega}_{N}^{(N)}\right)$, to be the closure in $H^{t}\left(\hat{\Omega}_{k}^{(q)}\right)$ of the smooth functions on $\hat{\Omega}_{k}^{(q)}$ that satisfy these boundary conditions. 

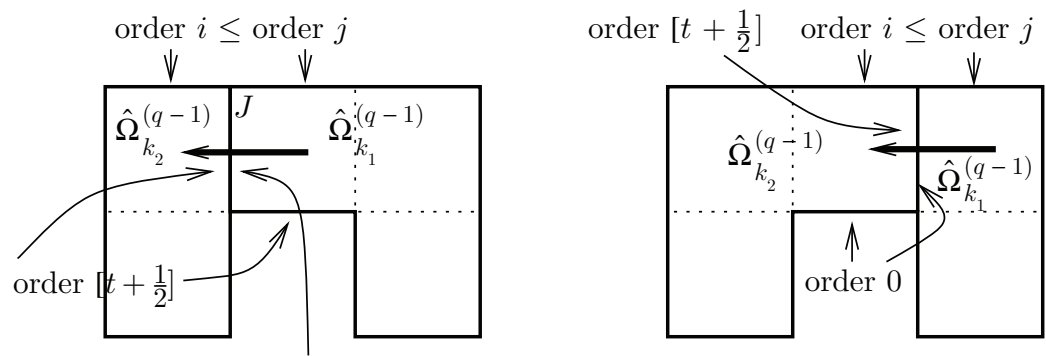

order 0

Figure 3. Two illustrations with $\left(\mathcal{D}_{1}-\mathcal{D}_{5}\right)$. The thick arrow indicates the action of the extension $E_{1}^{(q)}$.

Note that for $0 \leq k \leq N$, for some $\boldsymbol{\sigma}(k) \in\left(\left\{0, \ldots,\left\lfloor t+\frac{1}{2}\right\rfloor\right\}^{2}\right)^{n}$,

$$
\stackrel{\circ}{H}^{t}\left(\hat{\Omega}_{k}^{(0)}\right)=\stackrel{\circ}{H}{ }^{t}\left(\square_{k}\right)=H_{\boldsymbol{\sigma}(k)}^{t}\left(\square_{k}\right) .
$$

Remark 4.1. On the intersection of facets of hypercubes $\square_{k^{\prime}}$, the natural interpretation of the boundary conditions is the minimal one such that the boundary conditions on each of these facets is not violated.

The boundary conditions on the hypercubes that determine the spaces $\stackrel{\circ}{H}^{t}\left(\hat{\Omega}_{k}^{(q)}\right)$, and the order in which polytopes are joined should be chosen such that

$\left(\mathcal{D}_{4}\right)$ on the $\hat{\Omega}_{k_{1}}^{(q-1)}$ and $\hat{\Omega}_{k_{2}}^{(q-1)}$ sides of $J$, the boundary conditions are of order 0 and $\left\lfloor t+\frac{1}{2}\right\rfloor$, respectively,

and, w.l.o.g. assuming that $J=\{0\} \times \breve{J}$ and $(0,1) \times \breve{J} \subset \Omega_{k_{1}}^{(q-1)}$,

$\left(\mathcal{D}_{5}\right)$ for any function in $\stackrel{\circ}{H}^{t}\left(\hat{\Omega}_{k_{1}}^{(q-1)}\right)$ that vanishes near $\{0,1\} \times \breve{J}$, its reflection in $\{0\} \times \mathbb{R}^{n-1}$ (extended with zero, and then restricted to $\hat{\Omega}_{k_{2}}^{(q-1)}$ ) is in $\stackrel{\circ}{H}^{t}\left(\hat{\Omega}_{k_{2}}^{(q-1)}\right)$.

The condition $\left(\mathcal{D}_{5}\right.$ can be formulated by saying that the order of the boundary condition at any subfacet of $\hat{\Omega}_{k_{1}}^{(q-1)}$ adjacent to $J$ should not be less than this order at its reflection in $J$, where in case this reflection is not part of $\partial \hat{\Omega}_{k_{2}}^{(q-1)}$ the latter order should be read as the highest possible one $\left\lfloor t+\frac{1}{2}\right\rfloor$; and furthermore, that the order of the boundary condition at any subfacet of $\hat{\Omega}_{k_{2}}^{(q-1)}$ adjacent to $J$ should not be larger than this order at its reflection in $J$, where in case this reflection is not part of $\partial \hat{\Omega}_{k_{1}}^{(q-1)}$, the latter order should be read as the lowest possible one 0 . See Figure 3 for an illustration.

Given $1 \leq q \leq N$, for $i \in\{1,2\}$, let $R_{i}^{(q)}$ be the restriction of functions on $\hat{\Omega}_{\bar{k}}^{(q)}$ to $\hat{\Omega}_{k_{i}}^{(q-1)}$, let $\eta_{2}^{(q)}$ be the extension of functions on $\hat{\Omega}_{k_{2}}^{(q-1)}$ to $\hat{\Omega}_{\bar{k}}^{(q)}$ by zero, and let $E_{1}^{(q)}$ be some extension of functions on $\hat{\Omega}_{k_{1}}^{(q-1)}$ to $\hat{\Omega}_{\bar{k}}^{(q)}$.

Proposition 4.2. Assume that

$$
E_{1}^{(q)} \in B\left(L_{2}\left(\hat{\Omega}_{k_{1}}^{(q-1)}\right), L_{2}\left(\hat{\Omega}_{\bar{k}}^{(q)}\right)\right), \quad E_{1}^{(q)} \in B\left(\stackrel{\circ}{H}^{t}\left(\hat{\Omega}_{k_{1}}^{(q-1)}\right), \stackrel{\circ}{H}^{t}\left(\hat{\Omega}_{\bar{k}}^{(q)}\right)\right) .
$$


Then for $s \in[0,1]$,

$$
E^{(q)}:=\left[E_{1}^{(q)} \quad \eta_{2}^{(q)}\right] \in B\left(\prod_{i=1}^{2}\left[L_{2}\left(\hat{\Omega}_{k_{i}}^{(q-1)}\right), \stackrel{\circ}{H}{ }^{t}\left(\hat{\Omega}_{k_{i}}^{(q-1)}\right)\right]_{s, 2},\left[L_{2}\left(\hat{\Omega}_{\bar{k}}^{(q)}\right), \stackrel{\circ}{H^{t}}\left(\hat{\Omega}_{\bar{k}}^{(q)}\right)\right]_{s, 2}\right),
$$

is boundedly invertible.

Proof. Taking $V^{(q)}=L_{2}\left(\hat{\Omega}_{\bar{k}}^{(q)}\right), V_{i}^{(q)}=L_{2}\left(\hat{\Omega}_{k_{i}}^{(q-1)}\right), \underline{V}^{(q)}=\stackrel{\circ}{H}^{t}\left(\hat{\Omega}_{\bar{k}}^{(q)}\right), \underline{V}_{i}^{(q)}=$ $\stackrel{\circ}{H}^{t}\left(\hat{\Omega}_{k_{i}}^{(q-1)}\right)$, and noting that $\Im\left(\operatorname{Id}-E_{1}^{(q)} R_{1}^{(q)}\right) \subset\left\{u \in \stackrel{\circ}{H}^{t}\left(\hat{\Omega}_{\bar{k}}^{(q)}\right): u=0\right.$ on $\left.\hat{\Omega}_{k_{1}}^{(q-1)}\right\}=$ $\Im\left(\left.\eta_{2}^{(q)}\right|_{H^{t}\left(\hat{\Omega}_{k_{2}}^{(q-1)}\right)}\right)$, the result follows from an application of Proposition 2.5(a).

Corollary 4.3. For $E$ being the composition for $q=1, \ldots, N$ of the mappings $E^{(q)}$ from Proposition 4.2, trivially extended with identity operators in coordinates $k \in\{q-1, \ldots, N\} \backslash\left\{k_{1}^{(q)}, k_{2}^{(q)}\right\}$, it holds that

$$
E \in B\left(\prod_{k=0}^{n}\left[L_{2}\left(\square_{k}\right), \stackrel{\circ}{H}^{t}\left(\square_{k}\right)\right]_{s, 2},\left[L_{2}(\hat{\Omega}), \stackrel{\circ}{H}^{t}(\hat{\Omega})\right]_{s, 2}\right)
$$

is boundedly invertible.

Under the conditions $\left(\mathcal{D}_{1}-\mathcal{D}_{5}\right)$, the extensions $E_{1}^{(q)}$ can be constructed (essentially) as tensor products of univariate extensions with identity operators in the other Cartesian directions.

Proposition 4.4. W.l.o.g. let $J=\{0\} \times \breve{J}$ and $(0,1) \times \breve{J} \subset \hat{\Omega}_{k_{1}}^{(q-1)}$. Let $G_{1}$ be an extension operator of functions on $(0,1)$ to functions on $(-1,1)$ such that

$$
G_{1} \in B\left(L_{2}(0,1), L_{2}(-1,1)\right), \quad G_{1} \in B\left(H^{t}(0,1), H_{\left(\left\lfloor t+\frac{1}{2}\right\rfloor, 0\right)}^{t}(-1,1)\right) .
$$

Then $E_{1}^{(q)}$ defined by $R_{2}^{(q)} E_{1}^{(q)}$ being the composition of the restriction to $(0,1) \times \breve{J}$, followed by an application of

$$
G_{1} \otimes \mathrm{Id} \otimes \cdots \otimes \mathrm{Id},
$$

followed by an extension by 0 to $\hat{\Omega}_{k_{2}}^{(q-1)} \backslash(-1,0) \times \breve{J}$, satisfies the assumptions made in Proposition 4.2 .

Remark 4.5. The condition that an extension by $G_{1}$ vanishes up to order $\left\lfloor t+\frac{1}{2}\right\rfloor$ at -1 is fully harmless since it can easily be enforced by multiplying an extension by some smooth cut-off function. The scale-dependent extension that we will discuss in Subsection 5.1 satisfies this boundary condition automatically.

Our main interest of Corollary 4.3 lies in the following:

Corollary 4.6. For $0 \leq k \leq N$, let $\boldsymbol{\Psi}_{k}$ be a Riesz basis for $L_{2}\left(\square_{k}\right)$, that renormalized in $H^{t}\left(\square_{k}\right)$, is a Riesz basis for $H^{t}\left(\square_{k}\right)=H_{\boldsymbol{\sigma}(k)}^{t}(\square)$. Then for $s \in[0,1]$, and with $E$ from Corollary 4.3 , the collection $E\left(\prod_{k=0}^{N} \Psi_{k}\right)$, normalized in the corresponding norm, is a Riesz basis for $\left[L_{2}(\hat{\Omega}), \stackrel{\circ}{H}(\hat{\Omega})\right]_{s, 2}$.

Remark 4.7. Although we allow for $t \in\left(0, \frac{1}{2}\right)$, for these values of $t$ our exposition is not very relevant. Indeed, for those $t$, a piecewise tensor product basis can simply be constructed as the union of the tensor product bases on the hypercubes. 
To find the corresponding dual basis, we follow Section 2 . Taking for $q=$ $1, \ldots, N$,

$$
H^{(q)}=L_{2}\left(\hat{\Omega}_{\bar{k}}^{(q)}\right), \quad H_{i}^{(q)}=L_{2}\left(\hat{\Omega}_{k_{i}}^{(q)}\right),
$$

and with $\eta_{1}^{(q)}$ being the extension of functions on $\hat{\Omega}_{k_{1}}^{(q-1)}$ to $\hat{\Omega}_{\bar{k}}^{(q)}$ by zero, Proposition 2.2 shows that

$$
\left(E^{(q)}\right)^{-*}=\left[\eta_{1}^{(q)} \quad\left(\mathrm{Id}-\eta_{1}^{(q)}\left(E_{1}^{(q)}\right)^{*}\right) \eta_{2}^{(q)}\right] .
$$

Corollary 4.8. In the situation of Corollary 4.6, let $\tilde{\mathbf{\Psi}}_{k}$ the Riesz basis for $L_{2}\left(\square_{k}\right)$ that is dual to $\mathbf{\Psi}_{k}$. Then $E^{-*}\left(\prod_{k=0}^{N} \tilde{\mathbf{\Psi}}_{k}\right)$ is the Riesz basis for $L_{2}(\hat{\Omega})$ that is dual to $E\left(\prod_{k=0}^{N} \Psi_{k}\right)$. The operator $E^{-*}$ is the composition for $q=1, \ldots, N$ of the mappings $\left(E^{(q)}\right)^{-*}$ trivially extended with identity operators in coordinates $k \in$ $\{q-1, \ldots, N\} \backslash\left\{k_{1}^{(q)}, k_{2}^{(q)}\right\}$.

Below we give conditions such that $E^{-*}\left(\prod_{k=0}^{N} \tilde{\boldsymbol{\Psi}}_{k}\right)$, properly scaled, is a Riesz basis for a range of Sobolev spaces with positive smoothness indices, and so, equivalently, $E\left(\prod_{k=0}^{N} \Psi_{k}\right)$ to be a Riesz basis for the corresponding dual spaces.

For some $\tilde{t} \in[0, \infty) \backslash\left(\mathbb{N}_{0}+\left\{\frac{1}{2}\right\}\right)$, to each of the closed facets of all the hypercubes $\square_{k}$, we associate a number in $\left\{0, \ldots,\left\lfloor\tilde{t}+\frac{1}{2}\right\rfloor\right\}$ indicating the order of the dual Dirichlet boundary condition on that facet. On facets on the boundary of $\hat{\Omega}$, this number can be chosen arbitrarily, where on the interior facets it is 0 or $\left\lfloor\tilde{t}+\frac{1}{2}\right\rfloor$.

We define $\stackrel{\circ}{H}{ }^{\tilde{t}}\left(\hat{\Omega}_{k}^{(q)}\right)$, and so for $k=q=N$ in particular $\stackrel{\circ}{H}^{\tilde{t}}(\hat{\Omega})=\stackrel{\circ}{H}^{\tilde{t}}\left(\hat{\Omega}_{N}^{(N)}\right)$, to be the closure in $H^{\tilde{t}}\left(\hat{\Omega}_{k}^{(q)}\right)$ of the smooth functions on $\hat{\Omega}_{k}^{(q)}$ that on any of its facets satisfy the boundary conditions that were imposed on each of its subfacets. Note that with some abuse of notation, even when $\tilde{t}=t$ generally $\stackrel{\circ}{H}^{\tilde{t}}\left(\hat{\Omega}_{k}^{(q)}\right) \neq \stackrel{\stackrel{t}{H}}{H^{t}}\left(\hat{\Omega}_{k}^{(q)}\right)$, and that for $0 \leq k \leq N$,

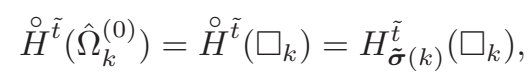

for some $\tilde{\boldsymbol{\sigma}}(k) \in\left(\left\{0, \ldots,\left\lfloor\tilde{t}+\frac{1}{2}\right\rfloor\right\}^{2}\right)^{n}$.

We make the following assumptions on the selection of the boundary conditions that determine the dual spaces $\stackrel{\circ}{H}^{\tilde{t}}\left(\hat{\Omega}_{k}^{(q)}\right)$ :

$\left(\mathcal{D}_{4}^{\prime}\right)$ on the $\hat{\Omega}_{k_{1}}^{(q-1)}$ and $\hat{\Omega}_{k_{2}}^{(q-1)}$ sides of $J$, the boundary conditions are of order $\left\lfloor\tilde{t}+\frac{1}{2}\right\rfloor$ and 0 , respectively,

and, w.l.o.g. assuming that $J=\{0\} \times \breve{J}$ and $(0,1) \times \breve{J} \subset \Omega_{k_{1}}^{(q-1)}$,

$\left(\mathcal{D}_{5}^{\prime}\right)$ for any function in $\stackrel{\circ}{H^{t}}\left(\hat{\Omega}_{k_{2}}^{(q-1)}\right)$ that vanishes near $\{-1,0\} \times \breve{J}$, its reflection in $\{0\} \times \mathbb{R}^{n-1}$ (extended with zero, and then restricted to $\hat{\Omega}_{k_{1}}^{(q-1)}$ ) is in $\stackrel{\circ}{H^{\tilde{t}}}\left(\hat{\Omega}_{k_{1}}^{(q-1)}\right)$.

Proposition 4.9. For $1 \leq q \leq N$, let the extension $E_{1}^{(q)}$ be of tensor product form as in Proposition 4.4 with $G_{1}^{*} \in B\left(H_{\left(0,\left\lfloor\tilde{t}+\frac{1}{2}\right\rfloor\right)}^{\tilde{t}}(-1,1), H_{\left(\left\lfloor\tilde{t}+\frac{1}{2}\right\rfloor,\left\lfloor\tilde{t}+\frac{1}{2}\right\rfloor\right)}^{\tilde{t}}(0,1)\right)$, and let $\tilde{\mathbf{\Psi}}_{k}$, properly scaled, be a Riesz basis for $\stackrel{\stackrel{t}{H}}{\tilde{t}}\left(\square_{k}\right)$. Then for $s \in[0,1], E^{-*}\left(\prod_{k=0}^{N} \tilde{\mathbf{\Psi}}_{k}\right)$ is, properly scaled, a Riesz basis for $\left[L_{2}(\hat{\Omega}), \stackrel{\circ}{H}{ }^{\tilde{t}}(\hat{\Omega})\right]_{s, 2}$.

Remark 4.10. The boundary conditions imposed on $G_{1}^{*} u$ at 1 are fully harmless. The scale-dependent extension $G_{1}$ that we will discuss in Subsection [5.1] satisfies 
these boundary conditions automatically. On the other hand, thinking of $t \geq \tilde{t}$, the boundary conditions at 0 are, when $\tilde{t}>\frac{1}{2}$, the only properties that are not already implied by the conditions on $G_{1}$ from Proposition 4.4

Proof. The conditions $\mathcal{D}_{4}^{\prime}, \mathcal{D}_{5}^{\prime}$ imply both that $R_{2}^{(q)}$ has a right-inverse which is in $B\left(\stackrel{\circ}{H}{ }^{\tilde{t}}\left(\Omega_{k_{2}}^{(q-1)}\right), \stackrel{\circ}{H} \tilde{t}^{(q)}\left(\Omega_{\bar{k}}^{(q)}\right)\right)$ and $\left(E_{1}^{(q)}\right)^{*} \in B\left(\stackrel{\circ}{H^{t}}\left(\Omega_{\bar{k}}^{(q)}\right), \stackrel{\circ}{H}^{\tilde{t}}\left(\Omega_{k_{1}}^{(q-1)}\right)\right)$, by the assumption on $G_{1}^{*}$. Since $R_{2}^{(q)} \in B\left(\stackrel{\circ}{H^{\tilde{t}}}\left(\Omega_{\bar{k}}^{(q)}\right), \stackrel{\circ}{H^{\tilde{t}}}\left(\Omega_{k_{2}}^{(q-1)}\right)\right), \eta_{1}^{(q)} \in B\left(\stackrel{\circ}{H^{\tilde{t}}}\left(\Omega_{k_{1}}^{(q-1)}\right), \stackrel{\circ}{H^{\tilde{t}}}\left(\Omega_{\bar{k}}^{(q)}\right)\right)$ directly follow from $\left(\mathcal{D}_{4}^{\prime}\right.$, an $N$-fold application of Proposition 2.4 together with the assumption on the bases $\tilde{\boldsymbol{\Psi}}_{k}$ completes the proof.

To end the discussion about the stability of $E\left(\Pi_{k=0}^{N} \boldsymbol{\Psi}_{k}\right)$ in dual norms, we note that for $\tilde{t}<\frac{1}{2}$, which suffices for our application for solving PDEs, the conditions $\left(\mathcal{D}_{4}^{\prime}, \mathcal{D}_{5}^{\prime}\right.$, and those from Proposition 4.9 are void, with the exception of the very mild condition of $\tilde{\boldsymbol{\Psi}}_{k}$, properly scaled, being a Riesz basis for $\stackrel{\circ}{H} \tilde{t}^{(}\left(\square_{k}\right)$.

The construction of Riesz bases on the reference domain $\hat{\Omega}$ extends to more general domains in a standard fashion. Let $\Omega$ be the image of $\hat{\Omega}$ under a homeomorphism $\kappa$. We define the pull-back $\kappa^{*}$ by $\kappa^{*} w=w \circ \kappa$, and so its inverse $\kappa^{-*}$, known as the push-forward, satisfies $\kappa^{-*} v=v \circ \kappa^{-1}$.

Proposition 4.11. Let $\kappa^{*}$ be boundedly invertible as a mapping both from $L_{2}(\Omega)$ to $L_{2}(\hat{\Omega})$ and from $H^{t}(\Omega)$ to $H^{t}(\hat{\Omega})$. Setting $\stackrel{\circ}{H}^{t}(\Omega):=\left.\Im \kappa^{-*}\right|_{H^{t}}{ }_{(\hat{\Omega})}$, we have that $\kappa^{-*} \in B\left(\left[L_{2}(\hat{\Omega}), \stackrel{\circ}{H}{ }^{t}(\hat{\Omega})\right]_{s, 2},\left[L_{2}(\Omega), \stackrel{\circ}{H}{ }^{t}(\Omega)\right]_{s, 2}\right)$ is boundedly invertible $(s \in[0,1])$. So if $\boldsymbol{\Psi}$ is a Riesz basis for $L_{2}(\hat{\Omega})$ and, properly scaled, for $\stackrel{\circ}{H}^{t}(\hat{\Omega})$, then for $s \in[0,1]$, $\kappa^{-*} \boldsymbol{\Psi}$ is, properly scaled, a Riesz basis for $\left[L_{2}(\Omega), \stackrel{\circ}{H}^{t}(\Omega)\right]_{s, 2}$.

If $\tilde{\boldsymbol{\Psi}}$ is the collection dual to $\boldsymbol{\Psi}$, then $\left|\operatorname{det} D \kappa^{-1}(\cdot)\right| \kappa^{-*} \tilde{\boldsymbol{\Psi}}$ is the collection dual to $\kappa^{-*} \mathbf{\Psi}$.

\section{Approximation by -Piecewise- tensor product Wavelets}

In the setting of Proposition 4.4, Corollary 4.6 and Proposition 4.9, writing $\square_{k}=\square+\alpha_{k}$, where $\alpha_{k} \in \mathbb{Z}^{n}$, we select the the primal and dual bases for $L_{2}\left(\square_{k}\right)$ to be

$$
\boldsymbol{\Psi}_{\boldsymbol{\sigma}(k), \tilde{\boldsymbol{\sigma}}(k)}\left(\cdot-\alpha_{k}\right), \quad \tilde{\boldsymbol{\Psi}}_{\boldsymbol{\sigma}(k), \tilde{\boldsymbol{\sigma}}(k)}\left(\cdot-\alpha_{k}\right)
$$

as constructed in Section 3, which, properly scaled, are Riesz bases for $H_{\boldsymbol{\sigma}(k)}^{t}\left(\square_{k}\right)$ and $H_{\tilde{\boldsymbol{\sigma}}(k)}^{\tilde{t}}\left(\square_{k}\right)$, respectively.

In the setting of Proposition 4.11, for $m \in\{0, \ldots,\lfloor t\rfloor\}$ and $u \in \stackrel{\circ}{H}^{m}(\Omega):=$ $\left[L_{2}(\Omega), \stackrel{\circ}{H}{ }^{t}(\Omega)\right]_{m / t, 2}$, with additionally

$$
u \in \kappa^{-*}\left(\prod_{k=0}^{N} \mathcal{H}_{m, \theta}^{d}\left(\square_{k}\right)\right):=\left\{v: \Omega \rightarrow \mathbb{R}: v \circ \kappa \in \prod_{k=0}^{N} \mathcal{H}_{m, \theta}^{d}\left(\square_{k}\right)\right\},
$$

we study approximation rates from $\kappa^{-*} E\left(\prod_{k=0}^{N} \Psi_{\boldsymbol{\sigma}(k), \tilde{\boldsymbol{\sigma}}(k)}\left(\cdot-\alpha_{k}\right)\right)$ in the $H^{m}(\Omega)$ norm. Since, as is assumed in Proposition 4.11, $\kappa^{*} \in B\left(\stackrel{\circ}{H^{m}}(\Omega), \stackrel{\circ}{H}^{m}(\hat{\Omega})\right)$ is boundedly invertible, it is sufficient to study this issue for the case that $\kappa=$ Id and so $\Omega=\hat{\Omega}$.

We will apply extension operators $E_{1}^{(q)}$ that are built from univariate extension operators. The latter will be chosen such that the resulting primal and dual wavelets on $\hat{\Omega}$ are, restricted to each $\square_{k} \subset \hat{\Omega}$, tensor products of collections of univariate 
functions that are local and locally finite (cf. parts (11) and (2) of the forthcoming Proposition 5.4).

5.1. Construction of scale-dependent extension operators. We make the following additional assumptions on the univariate wavelets. For $\vec{\sigma}=\left(\sigma_{\ell}, \sigma_{r}\right) \in$ $\left\{0, \ldots,\left\lfloor t+\frac{1}{2}\right\rfloor\right\}^{2}, \overrightarrow{\tilde{\sigma}}=\left(\tilde{\sigma}_{\ell}, \tilde{\sigma}_{r}\right) \in\left\{0, \ldots,\left\lfloor\tilde{t}+\frac{1}{2}\right\rfloor\right\}^{2}$, and with $\overrightarrow{0}:=(0,0)$,

$\left(\mathcal{W}_{7}\right) V_{j}^{(\vec{\sigma})}:=\operatorname{span}\left\{\psi_{\lambda}^{(\vec{\sigma}, \overrightarrow{\tilde{\sigma}})}: \lambda \in \nabla_{\vec{\sigma}, \vec{\sigma}},|\lambda| \leq j\right\}$ is independent of $\overrightarrow{\tilde{\sigma}}$, and $V_{j}^{(\vec{\sigma})}=$ $V_{j}^{(\overrightarrow{0})} \cap H_{\vec{\sigma}}^{t}(\mathcal{I})$,

$\left(\mathcal{W}_{8}\right) \nabla_{\vec{\sigma}, \overrightarrow{\tilde{\sigma}}}$ is the disjoint union of $\nabla_{\sigma_{\ell}, \tilde{\sigma}_{\ell}}^{(\ell)}, \nabla^{(I)}, \nabla_{\sigma_{r}, \tilde{\sigma}_{r}}^{(r)}$ such that

(i)

$$
\sup _{\lambda \in \nabla_{\vec{\sigma}, \vec{\sigma}}^{(\ell)}, x \in \operatorname{supp} \psi_{\lambda}^{(\vec{\sigma}, \vec{\sigma})}} 2^{|\lambda|}|x| \lesssim \rho, \sup _{\lambda \in \nabla_{\vec{\sigma}, \vec{\sigma}}^{(r)}, x \in \operatorname{supp} \psi_{\lambda}^{(\vec{\sigma}, \vec{\sigma})}} 2^{|\lambda|}|1-x| \lesssim \rho,
$$

(ii) for $\lambda \in \nabla^{(I)}, \psi_{\lambda}^{(\vec{\sigma}, \overrightarrow{\tilde{\sigma}})}=\psi_{\lambda}^{(\overrightarrow{0}, \overrightarrow{0})}, \tilde{\psi}_{\lambda}^{(\vec{\sigma}, \overrightarrow{\tilde{\sigma}})}=\tilde{\psi}_{\lambda}^{(\overrightarrow{0}, \overrightarrow{0})}$, and the extensions of $\psi_{\lambda}^{(\overrightarrow{0}, \overrightarrow{0})}$ and $\tilde{\psi}_{\lambda}^{(\overrightarrow{0}, \overrightarrow{0})}$ by zero are in $H^{t}(\mathbb{R})$ and $H^{\tilde{t}}(\mathbb{R})$, respectively.

$$
\begin{gathered}
\left(\mathcal{W}_{9}\right)\left\{\begin{array}{c}
\operatorname{span}\left\{\psi_{\lambda}^{(\overrightarrow{0}, \overrightarrow{0})}(1-\cdot): \lambda \in \nabla^{(I)},|\lambda|=j\right\}=\operatorname{span}\left\{\psi_{\lambda}^{(\overrightarrow{0}, \overrightarrow{0})}: \lambda \in \nabla^{(I)},|\lambda|=j\right\}, \\
\operatorname{span}\left\{\psi_{\lambda}^{\left(\sigma_{\ell}, \sigma_{r}\right),\left(\tilde{\sigma}_{\ell}, \tilde{\sigma}_{r}\right)}(1-\cdot): \lambda \in \nabla_{\sigma_{\ell}, \tilde{\sigma}_{\ell},}^{(\ell)}|\lambda|=j\right\} \\
=\operatorname{span}\left\{\psi_{\lambda}^{\left(\sigma_{r}, \sigma_{\ell}\right),\left(\tilde{\sigma}_{r}, \tilde{\sigma}_{\ell}\right)}: \lambda \in \nabla_{\left.\sigma_{r}, \tilde{\sigma}_{r},|\lambda|=j\right\},}^{(r)},|\lambda|\right.
\end{array}\right. \\
\left(\mathcal{W}_{10}\right)\left\{\begin{array}{l}
\psi_{\lambda}^{(\vec{\sigma}, \vec{\sigma})}\left(2^{l} \cdot\right) \in \operatorname{span}\left\{\psi_{\mu}^{(\vec{\sigma}, \vec{\sigma})}: \mu \in \nabla_{\sigma_{\ell}, \tilde{\sigma}_{\ell}}^{(\ell)}\right\} \quad\left(l \in \mathbb{N}_{0}, \lambda \in \nabla_{\left.\sigma_{\ell}, \tilde{\sigma}_{\ell}\right),}^{(\ell)}\right) \\
\psi_{\lambda}^{(\overrightarrow{0}, \overrightarrow{0})}\left(2^{l} \cdot\right) \in \operatorname{span}\left\{\psi_{\mu}^{(\overrightarrow{0}, \overrightarrow{0})}: \mu \in \nabla^{(I)}\right\} \quad\left(l \in \mathbb{N}_{0}, \lambda \in \nabla^{(I)}\right) .
\end{array}\right.
\end{gathered}
$$

As $\left(\mathcal{W}_{1}-\left(\mathcal{W}_{6}\right)\right.$, these conditions are satisfied by following the biorthogonal wavelet constructions on the interval from $\left[\right.$ Pri10, Dij09 $\left(\not \mathcal{W}_{7}\right)$ is not satisfied by the construction from DKU99, but the following exposition can be adapted to apply to these wavelets as well).

Remark 5.1. In view of the boundary conditions that are imposed on the interfacets (see $\left.\mathcal{D}_{4}\right)$ and $\left(\mathcal{D}_{4}^{\prime}\right)$ ) it is actually sufficient to impose $\left(\mathcal{W}_{7}-\left(\mathcal{W}_{10}\right)\right.$ for $\left(\sigma_{\ell}, \tilde{\sigma}_{\ell}\right),\left(\sigma_{r}, \tilde{\sigma}_{r}\right) \in\left\{\left(\left\lfloor t+\frac{1}{2}\right\rfloor, 0\right),\left(0,\left\lfloor\tilde{t}+\frac{1}{2}\right\rfloor\right)\right\}$.

We consider the setting of Proposition 4.4 W.l.o.g. we assume that $J=\{0\} \times \breve{J}$, and $(0,1) \times \breve{J} \subset \hat{\Omega}_{k_{1}}^{(q-1)}$. We assume to have available a univariate extension operator

$$
\breve{G}_{1} \in B\left(L_{2}(0,1), L_{2}(-1,1)\right) \text { with }\left\{\begin{array}{c}
\breve{G}_{1} \in B\left(H^{t}(0,1), H^{t}(-1,1)\right), \\
\breve{G}_{1}^{*} \in B\left(H^{\tilde{t}}(-1,1), H_{\left(\left\lfloor\tilde{t}+\frac{1}{2}\right\rfloor, 0\right)}^{(0,1))} .\right.
\end{array}\right.
$$

Let $\eta_{1}$ and $\eta_{2}$ denote the extensions by zero of functions on $(0,1)$ or on $(-1,0)$ to functions on $(-1,1)$, with $R_{1}$ and $R_{2}$ denoting their adjoints. We assume that $\breve{G}_{1}$ and its "adjoint extension"

$$
\breve{G}_{2}:=\left(\mathrm{Id}-\eta_{1} \breve{G}_{1}^{*}\right) \eta_{2}
$$

(cf. Remark 2.3) are local in the sense that

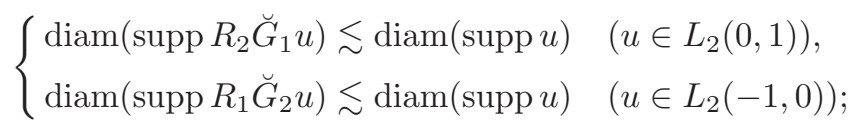

see Figure 4 for an illustration. 


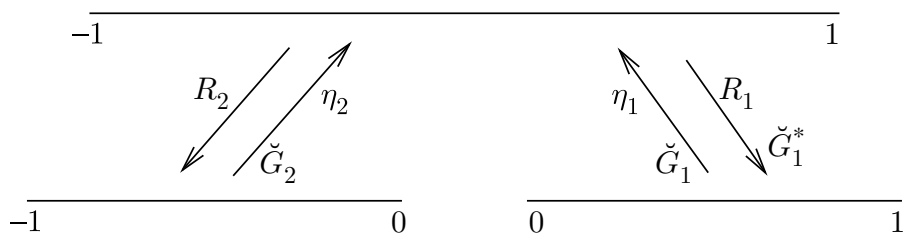

FiguRE 4. Univariate extensions and restrictions.

Examples are given by Hestenes extensions ([Hes41, DS99, KS06]), which are of the form

$$
\breve{G}_{1} v(-x)=\sum_{l=0}^{L} \gamma_{l}(\zeta v)\left(\beta_{l} x\right) \quad\left(v \in L_{2}(\mathcal{I}), x \in \mathcal{I}\right)
$$

(and, being an extension, $\breve{G}_{1} v(x)=v(x)$ for $x \in \mathcal{I}$ ), where $\gamma_{l} \in \mathbb{R}, \beta_{l}>0$, and $\zeta:[0, \infty) \rightarrow[0, \infty)$ is some smooth cut-off function with $\zeta \equiv 1$ in a neighborhood of 0 , and $\operatorname{supp} \zeta \subset\left[0, \min _{l}\left(\beta_{l}, \beta_{l}^{-1}\right)\right]$. Its adjoint reads as

$$
\breve{G}_{1}^{*} w(x)=w(x)+\zeta(x) \sum_{l=0}^{L} \frac{\gamma_{l}}{\beta_{l}} w\left(\frac{-x}{\beta_{l}}\right) \quad\left(w \in L_{2}(-1,1), x \in \mathcal{I}\right) .
$$

A Hestenes extension satisfies (5.2) if and only if

$$
\sum_{l=0}^{L} \gamma_{l} \beta_{l}^{i}=(-1)^{i}\left(\mathbb{N}_{0} \ni i \leq\left\lfloor t-\frac{1}{2}\right\rfloor\right), \sum_{l=0}^{L} \gamma_{l} \beta_{l}^{-(j+1)}=(-1)^{j+1}\left(\mathbb{N}_{0} \ni j \leq\left\lfloor\tilde{t}-\frac{1}{2}\right\rfloor\right) .
$$

With a univariate extension $\breve{G}_{1}$ as in (5.2) at hand, the obvious approach is to define $E_{1}^{(q)}$ according to Proposition 4.4 with $G_{1}=\breve{G}_{1}$. A problem with the choice $G_{1}=\breve{G}_{1}$ is that generally (5.3) does not imply the desirable property that $\operatorname{diam}\left(\operatorname{supp} G_{1} u\right) \lesssim \operatorname{diam}(\operatorname{supp} u)$. Indeed, think of the application of a Hestenes extension to a $u$ with a small support that is not located near the interface.

To solve this and the corresponding problem for the adjoint extension, in any case for $u$ being any primal or dual wavelet, respectively, following DS99. we will apply our construction using the modified, scale-dependent univariate extension operator

$$
G_{1}: u \mapsto \sum_{\lambda \in \nabla_{0,0}^{(\ell)}}\left\langle u, \tilde{\psi}_{\lambda}^{(\overrightarrow{0}, \overrightarrow{0})}\right\rangle_{L_{2}(\mathcal{I})} \breve{G}_{1} \psi_{\lambda}^{(\overrightarrow{0}, \overrightarrow{0})}+\sum_{\lambda \in \nabla^{(I)} \cup \nabla_{0,0}^{(r)}}\left\langle u, \tilde{\psi}_{\lambda}^{(\overrightarrow{0}, \overrightarrow{0})}\right\rangle_{L_{2}(\mathcal{I})} \eta_{1} \psi_{\lambda}^{(\overrightarrow{0}, \overrightarrow{0})}
$$

Taking $\breve{G}_{1}$ to be a Hestenes extension, under the condition of $\rho$ being sufficiently small, its first advantage is that its application in (5.5) does not "see" the cut-off function $\zeta$, which prevents potential quadrature problems.

Proposition 5.2. Assuming $\rho$ to be sufficiently small, the scale-dependent extension $G_{1}$ from (5.5) satisfies, for $\vec{\sigma} \in\left\{0, \ldots,\left\lfloor t+\frac{1}{2}\right\rfloor\right\}^{2}, \overrightarrow{\tilde{\sigma}} \in\left\{0, \ldots,\left\lfloor\tilde{t}+\frac{1}{2}\right\rfloor\right\}^{2}$,

$$
G_{1} \psi_{\mu}^{(\vec{\sigma}, \overrightarrow{\widetilde{\sigma}})}=\left\{\begin{array}{l}
\eta_{1} \psi_{\mu}^{(\vec{\sigma}, \overrightarrow{\tilde{\sigma}})} \text { when } \mu \in \nabla^{(I)} \cup \nabla_{\sigma_{r}, \tilde{\sigma}_{r}}^{(r)}, \\
\breve{G}_{1} \psi_{\mu}^{(\vec{\sigma}, \overrightarrow{\tilde{\sigma}})} \text { when } \mu \in \nabla_{\sigma_{\ell}, \tilde{\sigma}_{\ell}}^{(\ell)}
\end{array}\right.
$$


Assuming, additionally, $\breve{G}_{1}$ to be a Hestenes extension with $\beta_{l}=2^{l}$, the resulting adjoint extension $G_{2}:=\left(\mathrm{Id}-\eta_{1} G_{1}^{*}\right) \eta_{2}$ satisfies

$$
G_{2}\left(\tilde{\psi}_{\mu}^{(\vec{\sigma}, \overrightarrow{\tilde{\sigma}})}(1+\cdot)\right)=\left\{\begin{array}{l}
\eta_{2}\left(\tilde{\psi}_{\mu}^{(\vec{\sigma}, \overrightarrow{\tilde{\sigma}})}(1+\cdot)\right) \text { when } \mu \in \nabla^{(I)} \cup \nabla_{\sigma_{\ell}, \tilde{\sigma}_{\ell}}^{(\ell)} \\
\breve{G}_{2}\left(\tilde{\psi}_{\mu}^{(\vec{\sigma}, \overrightarrow{\tilde{\sigma}})}(1+\cdot)\right) \text { when } \mu \in \nabla_{\sigma_{r}, \tilde{\sigma}_{r}}^{(r)}
\end{array}\right.
$$

We have $G_{1} \in B\left(L_{2}(0,1), L_{2}(-1,1)\right), G_{1} \in B\left(H^{t}(0,1), H^{t}(-1,1)\right)$, and $G_{1}^{*} \in$ $B\left(H^{\tilde{t}}(-1,1), H_{\left(\left\lfloor\tilde{t}+\frac{1}{2}\right\rfloor, 0\right)}^{\tilde{t}}(0,1)\right)$.

Finally, for $\mu \in \nabla_{\vec{\sigma}, \vec{\sigma}}$, it holds that

$$
\begin{aligned}
\operatorname{diam}\left(\operatorname{supp} G_{1} \psi_{\mu}^{(\vec{\sigma}, \overrightarrow{\tilde{\sigma}})}\right) & \lesssim \operatorname{diam}\left(\operatorname{supp} \psi_{\mu}^{(\vec{\sigma}, \overrightarrow{\tilde{\sigma}})}\right), \\
\operatorname{diam}\left(\operatorname{supp} G_{2} \tilde{\psi}_{\mu}^{(\vec{\sigma}, \vec{\sigma})}(1+\cdot)\right) & \lesssim \operatorname{diam}\left(\operatorname{supp} \tilde{\psi}_{\mu}^{(\vec{\sigma}, \vec{\sigma})}(1+\cdot)\right) .
\end{aligned}
$$

Proof. By $\left(\mathcal{W}_{8}\right)$ (ii), for $\mu \in \nabla^{(I)} \cup \nabla_{\sigma_{r}, \tilde{\sigma}_{r}}^{(r)}, \lambda \in \nabla_{0,0}^{(\ell)}$, one has $\left\langle\psi_{\mu}^{(\vec{\sigma}, \overrightarrow{\tilde{\sigma}})}, \tilde{\psi}_{\lambda}^{(\overrightarrow{0}, \overrightarrow{0})}\right\rangle_{L_{2}(\mathcal{I})}=0$, and so $G_{1} \psi_{\mu}^{(\vec{\sigma}, \overrightarrow{\tilde{\sigma}})}=\sum_{\lambda \in \nabla_{\overrightarrow{0}, \overrightarrow{0}}}\left\langle\psi_{\mu}^{(\vec{\sigma}, \overrightarrow{\tilde{\sigma}})}, \tilde{\psi}_{\lambda}^{(\overrightarrow{0}, \overrightarrow{0})}\right\rangle_{L_{2}(\mathcal{I})} \eta_{1} \psi_{\lambda}^{(\overrightarrow{0}, \overrightarrow{0})}=\eta_{1} \psi_{\mu}^{(\vec{\sigma}, \overrightarrow{\tilde{\sigma}})}$, the last equality from $\Psi^{(\overrightarrow{0}, \overrightarrow{0})}$ being a Riesz basis for $L_{2}(\mathcal{I})$, and $\eta_{1}$ being $L_{2}$-bounded.

Similarly, for $\mu \in \nabla_{\sigma_{\ell}, \tilde{\sigma}_{\ell}}^{(\ell)}, \lambda \in \nabla^{(I)} \cup \nabla_{0,0}^{(r)}$, it holds that $\left\langle\psi_{\mu}^{(\vec{\sigma}, \overrightarrow{\tilde{\sigma}})}, \tilde{\psi}_{\lambda}^{(\overrightarrow{0}, \overrightarrow{0})}\right\rangle_{L_{2}(\mathcal{I})}=0$, and so $G_{1} \psi_{\mu}^{(\vec{\sigma}, \vec{\sigma})}=\sum_{\lambda \in \nabla_{(\overrightarrow{0}, \overrightarrow{0})}}\left\langle\psi_{\mu}^{(\vec{\sigma}, \vec{\sigma})}, \tilde{\psi}_{\lambda}^{(\overrightarrow{0}, \overrightarrow{0})}\right\rangle_{L_{2}(\mathcal{I})} \breve{G}_{1} \psi_{\lambda}^{(\overrightarrow{0}, \overrightarrow{0})}=\breve{G}_{1} \psi_{\mu}^{(\vec{\sigma}, \vec{\sigma})}$.

If $\breve{G}_{1}$ is a Hestenes extension with $\beta_{l}=2^{l}$, then for $v \in L_{2}(\mathcal{I})$,

$$
\begin{aligned}
G_{1}^{*} \eta_{2}(v(1+\cdot)) & =\sum_{\lambda \in \nabla_{\overrightarrow{0}, \overrightarrow{0}}}\left\langle G_{1}^{*} \eta_{2}(v(1+\cdot)), \psi_{\lambda}^{(\overrightarrow{0}, \overrightarrow{0})}\right\rangle_{L_{2}(\mathcal{I})} \tilde{\psi}_{\lambda}^{(\overrightarrow{0}, \overrightarrow{0})} \\
& =\sum_{\lambda \in \nabla_{\overrightarrow{0}, \overrightarrow{0}}}\left\langle v(1-\cdot),\left(R_{2} G_{1} \psi_{\lambda}^{(\overrightarrow{0}, \overrightarrow{0})}\right)(-\cdot)\right\rangle_{L_{2}(\mathcal{I})} \tilde{\psi}_{\lambda}^{(\overrightarrow{0}, \overrightarrow{0})} \\
& =\sum_{\lambda \in \nabla_{0,0}^{(\ell)}}\left\langle v(1-\cdot),\left(R_{2} \breve{G}_{1} \psi_{\lambda}^{(\overrightarrow{0}, \overrightarrow{0})}\right)(-\cdot)\right\rangle_{L_{2}(\mathcal{I})} \tilde{\psi}_{\lambda}^{(\overrightarrow{0}, \overrightarrow{0})} \\
& =\sum_{\lambda \in \nabla_{0,0}^{(\ell)}}\left\langle v(1-\cdot), \sum_{l=0}^{L} \gamma_{l} \psi_{\lambda}^{(\overrightarrow{0}, \overrightarrow{0})}\left(2^{l} \cdot\right)\right\rangle_{L_{2}(\mathcal{I})} \tilde{\psi}_{\lambda}^{(\overrightarrow{0}, \overrightarrow{0})} .
\end{aligned}
$$

For $v=\tilde{\psi}_{\mu}^{(\vec{\sigma}, \vec{\sigma})}$ and $\mu \in \nabla^{(I)} \cup \nabla_{\sigma_{\ell}, \tilde{\sigma}_{\ell}}^{(\ell)}$, (5.9) is zero by $\left(\mathcal{W}_{9}\right),\left(\mathcal{W}_{10}\right)$, and $\left(\mathcal{W}_{8}\right)$ (ii). For $v=\tilde{\psi}_{\mu}^{(\vec{\sigma}, \overrightarrow{\tilde{\sigma}})}$ and $\mu \in \nabla_{\sigma_{r}, \tilde{\sigma}_{r}}^{(r)}$, one has $\left\langle v(1-\cdot), \sum_{l=0}^{L} \gamma_{l}\left(\zeta \psi_{\lambda}^{(\overrightarrow{0}, \overrightarrow{0})}\right)\left(2^{l} \cdot\right)\right\rangle_{L_{2}(\mathcal{I})}=$ 0 for $\lambda \in \nabla^{(I)} \cup \nabla_{0,0}^{(r)}$ by $\left(\mathcal{W}_{9}\right),\left(\mathcal{W}_{10}\right)$, and $\left(\mathcal{W}_{8}\right)$ (ii). So for those $\mu$, one has $G_{1}^{*} \eta_{2} \tilde{\psi}_{\mu}^{(\vec{\sigma}, \vec{\sigma})}(1+\cdot)=\breve{G}_{1}^{*} \eta_{2} \tilde{\psi}_{\mu}^{(\vec{\sigma}, \overrightarrow{\tilde{\sigma}})}(1+\cdot)$, which completes the proof of (5.7).

Since $\operatorname{span}\left\{\psi_{\mu}^{(\overrightarrow{0}, \overrightarrow{0})}: \mu \in \nabla^{(I)} \cup \nabla_{0,0}^{(r)}\right\}+\operatorname{span}\left\{\psi_{\mu}^{(\overrightarrow{0}, \overrightarrow{0})}: \mu \in \nabla_{0,0}^{(\ell)}\right\}$ defines a stable splitting of both $L_{2}(\mathcal{I})$ and $H^{t}(\mathcal{I})$ into two subspaces, the statements about the boundedness of $G_{1}$ follow from (5.6) with $(\vec{\sigma}, \overrightarrow{\tilde{\sigma}})=(\overrightarrow{0}, \overrightarrow{0})$, (15.2), and $\left(\overrightarrow{\left.\mathcal{W}_{8}\right)}\right.$ (iii).

The mapping $P: u \mapsto \sum_{\mu \in \nabla^{(I)} \cup \nabla_{\sigma_{\ell}, \tilde{\sigma}_{\ell}}^{(\ell)}}\left\langle u, \psi_{\mu}^{(\vec{\sigma}, \overrightarrow{\tilde{\sigma}})}(1+\cdot)\right\rangle_{L_{2}(-1,0)} \eta_{2}\left(\tilde{\psi}_{\mu}^{(\vec{\sigma}, \overrightarrow{\widetilde{\sigma}})}(1+\cdot)\right)$ is in $B\left(H^{\tilde{t}}(-1,1), H^{\tilde{t}}(-1,1)\right)$ by the assumption on $\tilde{\Psi}^{(\vec{\sigma}, \vec{\sigma})}$ and $\left(\mathcal{W}_{8}\right)$ (ii). Since 
$\Psi^{(\vec{\sigma}, \vec{\sigma})}(1+\cdot)$ is a Riesz basis for $L_{2}(-1,0)$,

$$
R_{2}(I-P) u=\sum_{\mu \in \nabla_{\sigma_{r}, \tilde{\sigma}_{r}}^{(r)}}\left\langle u, \psi_{\mu}^{(\vec{\sigma}, \overrightarrow{\tilde{\sigma}})}(1+\cdot)\right\rangle_{L_{2}(-1,0)} \tilde{\psi}_{\mu}^{(\vec{\sigma}, \overrightarrow{\tilde{\sigma}})}(1+\cdot)
$$

We conclude that $\left(G_{1}^{*}-\breve{G}_{1}^{*}\right) \eta_{2} R_{2}(I-P)=0$. Since $G_{1}$ and $\breve{G}_{1}$ are extensions, we also have $G_{1}^{*} \eta_{1}=\operatorname{Id}=\breve{G}-1^{*} \eta_{1}$, and so $G_{1}^{*}(I-P)=G_{1}^{*}\left(\eta_{1} R_{1}+\eta_{2} R_{2}\right)(I-$ $P)=\breve{G}_{1}^{*}(I-P)$. Together with $G_{1}^{*} P=0$, from (5.2) we conclude that $G_{1}^{*} \in$ $B\left(H^{\tilde{t}}(-1,1), H_{\left(\left\lfloor\tilde{t}+\frac{1}{2}\right\rfloor, 0\right)}^{\tilde{t}}(0,1)\right)$.

The last statement is a direct consequence of (5.6) and (5.7).

Remark 5.3. Although implicitly claimed otherwise in [DS99, (4.3.12)], we note that (5.7), and so (5.8) cannot be expected for $\breve{G}_{1}$ being a general Hestenes extension as given by (5.4), without assuming that $\beta_{l}=2^{l}$.

Moreover, (5.7), and also (5.8), are only guaranteed when, for $\left(\sigma_{\ell}, \tilde{\sigma}_{\ell}\right)=(0,\lfloor\tilde{t}+$ $\left.\left.\frac{1}{2}\right\rfloor\right)$, for any $\lambda \in \nabla_{\vec{\sigma}, \vec{\sigma}}$ for which either $\psi_{\lambda}^{(\vec{\sigma}, \overrightarrow{\tilde{\sigma}})}$ or $\tilde{\psi}_{\lambda}^{(\vec{\sigma}, \overrightarrow{\tilde{\sigma}})}$ "depends on" the boundary conditions imposed at the left boundary, the primal wavelet $\psi_{\lambda}^{(\vec{\sigma}, \overrightarrow{\tilde{\sigma}})}$ is extended by the application of $\breve{G}_{1}$. The reason to emphasize this is that with common biorthogonal wavelet constructions on the interval, the number of dual wavelets that depend on the boundary conditions is larger than that of the primal ones. Note that even if dual wavelets may not enter the computations, their locality as given by (5.8) will be used to prove the forthcoming Theorem [5.6 about the approximation rates provided by the primal piecewise tensor product wavelets.

Some examples of relevant Hestenes extensions with $\beta_{l}=2^{l}$ are:

- $L=0, \gamma_{0}=1$ (reflection). Satisfies (5.2) for $t<\frac{3}{2}, \tilde{t}<\frac{1}{2}$.

- $L=1, \gamma_{0}=3, \gamma_{1}=-2$. Satisfies (5.2) for $t<\frac{5}{2}, \tilde{t}<\frac{1}{2}$.

- $L=1, \gamma_{0}=-3, \gamma_{1}=4$. Satisfies (5.2) for $t<\frac{3}{2}, \tilde{t}<\frac{3}{2}$.

- $L=2, \gamma_{0}=-5, \gamma_{1}=10, \gamma_{2}=-4$. Satisfies (5.2) for $t<\frac{5}{2}, \tilde{t}<\frac{3}{2}$.

In order to identify individual wavelets from the collections constructed by the applications of the extension operators, we have to introduce some more notation. For $0 \leq q \leq N$, we set the index sets

$$
\begin{aligned}
& \nabla_{k}^{(0)}:=\nabla_{\boldsymbol{\sigma}(k), \tilde{\boldsymbol{\sigma}}(k)} \times\{k\} \text { and, for } q>0, \\
& \boldsymbol{\nabla}_{k}^{(q)}:= \begin{cases}\nabla_{k_{1}}^{(q-1)} \cup \nabla_{k_{2}}^{(q-1)} & \text { if } k=\bar{k}, \\
\nabla_{\hat{k}}^{(q-1)} & \text { if } k \in\{q, \ldots, N\} \backslash\{\bar{k}\} \text { and } \Omega_{k}^{(q)}=\Omega_{\hat{k}}^{(q-1)},\end{cases}
\end{aligned}
$$


and, for $(\lambda, p) \in \nabla_{k}^{(q)}$, the primal and dual wavelets,

$$
\boldsymbol{\psi}_{\boldsymbol{\lambda}, p}^{(0, k)}:=\boldsymbol{\psi}_{\boldsymbol{\lambda}}^{(\boldsymbol{\sigma}(p), \tilde{\boldsymbol{\sigma}}(p))}\left(\cdot-\alpha_{p}\right), \quad \tilde{\boldsymbol{\psi}}_{\boldsymbol{\lambda}, p}^{(0, k)}:=\tilde{\boldsymbol{\psi}}_{\boldsymbol{\lambda}}^{(\boldsymbol{\sigma}(p), \tilde{\boldsymbol{\sigma}}(p))}\left(\cdot-\alpha_{p}\right),
$$

and, for $q>0$,

$$
\begin{aligned}
& \boldsymbol{\psi}_{\boldsymbol{\lambda}, p}^{(q, k)}:= \begin{cases}\left\{\begin{array}{ll}
E_{1}^{(q)} \boldsymbol{\psi}_{\boldsymbol{\lambda}, p}^{\left(q-1, k_{1}\right)} & (\boldsymbol{\lambda}, p) \in \boldsymbol{\nabla}_{k_{1}}^{(q-1)} \\
\eta_{2}^{(q)} \boldsymbol{\psi}_{\boldsymbol{\lambda}, p}^{\left(q-1, k_{2}\right)} & (\boldsymbol{\lambda}, p) \in \boldsymbol{\nabla}_{k_{2}}^{(q-1)}
\end{array}\right\} \quad \text { if } k=\bar{k}, \\
\boldsymbol{\psi}_{\boldsymbol{\lambda}, p}^{(q-1, \hat{k})} & \text { if } k \in\{q, \ldots, N\} \backslash\{\bar{k}\} \text { and } \Omega_{k}^{(q)}=\Omega_{\hat{k}}^{(q-1)},\end{cases}
\end{aligned}
$$

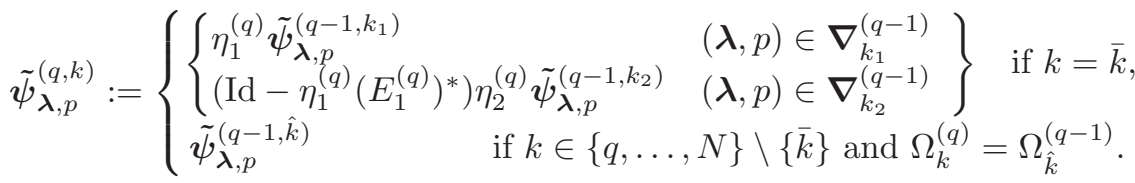

Then, as we have seen,

$$
\left.\left(\boldsymbol{\Psi}_{k}^{(q)}, \tilde{\boldsymbol{\Psi}}_{k}^{(q)}\right):=\left(\left\{\boldsymbol{\psi}_{\boldsymbol{\lambda}, p}^{(q, k)}\right):(\boldsymbol{\lambda}, p) \in \boldsymbol{\nabla}_{k}^{(q)}\right\},\left\{\tilde{\boldsymbol{\psi}}_{\boldsymbol{\lambda}, p}^{(q, k)}:(\boldsymbol{\lambda}, p) \in \boldsymbol{\nabla}_{k}^{(q)}\right\}\right)
$$

is a pair of biorthogonal Riesz bases for $L_{2}\left(\hat{\Omega}_{k}^{(q)}\right)$, and for $s \in[0,1], \Psi_{k}^{(q)}$ or $\tilde{\boldsymbol{\Psi}}_{k}^{(q)}$ are, properly scaled, Riesz bases for $\left[L_{2}\left(\hat{\Omega}_{k}^{(q)}\right), \stackrel{\stackrel{h}{H}}{t}\left(\hat{\Omega}_{k}^{(q)}\right)\right]_{s, 2}$ and $\left[L_{2}\left(\hat{\Omega}_{k}^{(q)}\right), \stackrel{\stackrel{\circ}{t}}{t}\left(\hat{\Omega}_{k}^{(q)}\right)\right]_{s, 2}$, respectively.

Proposition 5.4. With $E_{1}^{(q)}$ being defined using the scale-dependent extension operator as in Proposition 5.2, for $0 \leq q \leq k \leq N$, we have

(1) $\operatorname{supp} \boldsymbol{\psi}_{\boldsymbol{\lambda}, p}^{(q, k)}, \operatorname{supp} \tilde{\boldsymbol{\psi}}_{\boldsymbol{\lambda}, p}^{(q, k)}$ are contained in a hyperrectangle aligned with the Cartesian coordinates with sides in length of order $2^{-|\boldsymbol{\lambda}|_{1}}, \ldots, 2^{-|\boldsymbol{\lambda}|_{n}}$,

(2) for any $y \in \mathbb{R}^{n}$ and $\boldsymbol{j} \in \mathbb{N}_{0}^{n}$, the hyper-rectangle $y+\prod_{i=1}^{n}\left[0,2^{-j_{i}}\right]$ is intersected by the supports at most a uniformly bounded number of primal or dual wavelets $\boldsymbol{\psi}_{\boldsymbol{\lambda}, p}^{(q, k)}, \tilde{\boldsymbol{\psi}}_{\boldsymbol{\lambda}, p}^{(q, k)}$ with $|\boldsymbol{\lambda}|=\boldsymbol{j}$,

(3) let

$$
\begin{aligned}
& V_{\mathbf{j}}\left(\hat{\Omega}_{k}^{(q)}\right):=\left\{u \in \stackrel{\circ}{H}^{t}\left(\hat{\Omega}_{k}^{(q)}\right):\left.u\left(\cdot+\alpha_{k^{\prime}}\right)\right|_{\square} \in \bigotimes_{i=1}^{n} V_{j_{i}}^{(\overrightarrow{0})}\left(\square_{k^{\prime}} \subset \hat{\Omega}_{k}^{(q)}\right)\right\} \\
& Z_{\mathbf{j}}\left(\hat{\Omega}_{k}^{(q)}\right):=\operatorname{span}\left\{\boldsymbol{\psi}_{\boldsymbol{\lambda}, p}^{(q, k)}:(\boldsymbol{\lambda}, p) \in \nabla_{k}^{(q)},|\boldsymbol{\lambda}| \leq \boldsymbol{j}\right\},
\end{aligned}
$$

and $\mathbf{e}:=(1, \ldots, 1)^{\top} \in \mathbb{R}^{n}$. Then for some constants $m_{q}, M_{q} \in \mathbb{N}_{0}$, for all $\mathbf{j} \in\left\{m_{q}, m_{q}+1, \ldots\right\}^{n}$,

$$
V_{\mathbf{j}-m_{q}} \mathbf{e}\left(\hat{\Omega}_{k}^{(q)}\right) \subset Z_{\mathbf{j}}\left(\hat{\Omega}_{k}^{(q)}\right) \subset V_{\mathbf{j}+M_{q}} \mathbf{e}\left(\hat{\Omega}_{k}^{(q)}\right) .
$$

Proof. Parts (11) and (2) follow from the locality and the locally finiteness of the univariate primal and dual wavelets $\left(\left(\mathcal{W}_{5}\right)\right.$ and $\left(\mathcal{W}_{6}\right)$ ), and the locality of the extension and the adjoint extension given by (5.6) and (5.7).

By construction of the wavelet basis, the second inclusion in (3) follows from (5.6) and $\breve{G}_{1}$ being a Hestenes extension with $\beta_{l}=2^{l},\left(\mathcal{W}_{10}\right),\left(\mathcal{W}_{9}\right)$, and $\left(\mathcal{W}_{7}\right)$. The constant $M_{q}$ can be taken to be less than or equal to $2 L$, or to $L$ when the domain has no cracks.

The first inclusion in (3) holds true for $q=0$ with $m_{0}=0$ by (극 . Suppose, for some $m_{q-1}$, it is true for $q-1$ and $q-1 \leq k \leq N$. For some constant $m_{q} \geq m_{q-1}$ that 
will be determined below, let $v \in V_{\mathbf{j}-m_{q}} \mathbf{e}\left(\hat{\Omega}_{\bar{k}}^{(q)}\right)$. Then $R_{1}^{(q)} v \in V_{\mathbf{j}-m_{q}} \mathbf{e}\left(\hat{\Omega}_{k_{1}}^{(q-1)}\right) \subset$ $Z_{\mathbf{j}+\left(m_{q-1}-m_{q}\right) \mathbf{e}}\left(\hat{\Omega}_{k_{1}}^{(q-1)}\right)$, and so

$$
E_{1}^{(q)} R_{1}^{(q)} v \in Z_{\mathbf{j}+\left(m_{q-1}-m_{q}\right) \mathbf{e}}\left(\hat{\Omega}_{\bar{k}}^{(q)}\right) \subset Z_{\mathbf{j}}\left(\hat{\Omega}_{\bar{k}}^{(q)}\right)
$$

by definition of $\boldsymbol{\Psi}_{\bar{k}}^{(q)}$.

From (5.10), we have $E_{1}^{(q)} R_{1}^{(q)} v \in V_{\mathbf{j}+\left(m_{q-1}+M_{q}-m_{q}\right) \mathbf{e}}\left(\hat{\Omega}_{\bar{k}}^{(q)}\right)$, and so

$$
\left(I-E_{1}^{(q)} R_{1}^{(q)}\right) v \in V_{\mathbf{j}+\left(m_{q-1}+M_{q}-m_{q}\right) \mathbf{e}}\left(\hat{\Omega}_{\bar{k}}^{(q)}\right),
$$

and therefore

$$
\begin{aligned}
& R_{2}^{(q)}\left(\operatorname{Id}-E_{1}^{(q)} R_{1}^{(q)}\right) v \\
& \quad \in\left\{u \in L_{2}\left(\hat{\Omega}_{k_{2}}^{(q-1)}\right):\left.u\left(\cdot+\alpha_{k^{\prime}}\right)\right|_{\square} \in \bigotimes_{i=1}^{n} V_{j_{i}+m_{q-1}+M_{q}-m_{q}}^{(\overrightarrow{0})}\left(\square_{k^{\prime}} \subset \hat{\Omega}_{k_{2}}^{(q-1)}\right)\right\} .
\end{aligned}
$$

Since, as shown in the proof of Proposition 4.2, $\left(\operatorname{Id}-E_{1}^{(q)} R_{1}^{(q)}\right) v \in \Im\left(\left.\eta_{2}^{(q)}\right|_{H^{t}\left(\hat{\Omega}_{k_{2}}^{(q-1)}\right)}\right)$, and so $R_{2}^{(q)}\left(\operatorname{Id}-E_{1}^{(q)} R_{1}^{(q)}\right) v \in \stackrel{\circ}{H}^{t}\left(\hat{\Omega}_{k_{2}}^{(q-1)}\right)$, we infer that $R_{2}^{(q)}\left(\operatorname{Id}-E_{1}^{(q)} R_{1}^{(q)}\right) v \in$ $V_{\mathbf{j}+\left(m_{q-1}+M_{q}-m_{q}\right) \mathbf{e}}\left(\hat{\Omega}_{k_{2}}^{(q-1)}\right) \subset Z_{\mathbf{j}+\left(2 m_{q-1}+M_{q}-m_{q}\right) \mathbf{e}}\left(\hat{\Omega}_{k_{2}}^{(q-1)}\right)$. By taking $m_{q}=2 m_{q-1}+$ $M_{q}$, we conclude that $\left(\operatorname{Id}-E_{1}^{(q)} R_{1}^{(q)}\right) v=\eta_{2}^{(q)} R_{2}^{(q)}\left(\operatorname{Id}-E_{1}^{(q)} R_{1}^{(q)}\right) v \in Z_{\mathbf{j}}\left(\hat{\Omega}_{\bar{k}}^{(q)}\right)$ by definition of $\Psi_{\bar{k}}^{(q)}$. Together with (5.10), this completes the proof.

Remark 5.5. The above proof shows that for $L=0$ (reflection), $V_{\mathbf{j}}\left(\hat{\Omega}_{k}^{(q)}\right)=Z_{\mathbf{j}}\left(\hat{\Omega}_{k}^{(q)}\right)$.

Now we are ready to study the question, raised at the beginning of this section, about the rate of approximation in $\stackrel{\circ}{H}^{m}(\hat{\Omega})$ from the span of $\boldsymbol{\Psi}:=\mathbf{\Psi}_{N}^{(N)}$.

Theorem 5.6. Let the $E_{1}^{(q)}$ be defined using the scale-dependent extension operators as in Proposition 5.2. Then for any $\theta \in[0, d)$, and any $0 \leq q \leq k \leq N$, there exists a (nested) sequence $\left(\nabla_{k, M}^{(q)}\right)_{M \in \mathbb{N}} \subset \boldsymbol{\nabla}_{k}^{(q)}$ with $\# \boldsymbol{\nabla}_{k, M}^{(q)} \bar{\sim} M$, such that

$$
\inf _{v \in \operatorname{span}\left\{\boldsymbol{\psi}_{\boldsymbol{\lambda}, p}^{(q, k)}:(\boldsymbol{\lambda}, p) \in \nabla_{k, M}^{(q)}\right\}}\|u-v\|_{H^{m}\left(\hat{\Omega}_{k}^{(q)}\right)} \lesssim M^{-(d-m)} \sqrt{\left.\sum_{\square_{k^{\prime}} \subset \hat{\Omega}_{k}^{(q)}}\|u\|_{\mathcal{H}_{m, \theta}^{d}\left(\square_{k}^{\prime}\right)}^{2}\right)}
$$

for any $u \in \stackrel{\circ}{H}^{m}\left(\hat{\Omega}_{k}^{(q)}\right)$ for which the right-hand side is finite (for $q=k=N$, i.e., for $\hat{\Omega}_{k}^{(q)}=\hat{\Omega}$, this is equivalent to saying that u satisfies (5.1) $($ with $\left.\kappa=\mathrm{Id})\right)$.

For $m=0$, the factor $M^{-(d-m)}$ in (5.11) has to be read as $(\log M)^{(n-1)\left(\frac{1}{2}+d\right)} M^{-d}$.

Proof. We prove the statement with the additional property that the index sets $\nabla_{k, M}^{(q)}$ have the multiple tree property introduced in Theorem 3.2 for subsets of $\boldsymbol{\nabla}_{(\boldsymbol{\sigma}, \tilde{\boldsymbol{\sigma}})}$, and that in the current generalized setting reads as: For any $(\boldsymbol{\lambda}, p) \in \boldsymbol{\nabla}_{k, M}^{(q)}$ and any $\boldsymbol{j} \in \mathbb{N}_{0}^{n}$ with $\boldsymbol{j} \leq|\boldsymbol{\lambda}|$, there exists a $\left(\boldsymbol{\lambda}^{\prime}, p^{\prime}\right) \in \nabla_{k, M}^{(q)}$ with $\left|\boldsymbol{\lambda}^{\prime}\right|=\boldsymbol{j}$, and $\operatorname{supp} \boldsymbol{\psi}_{\boldsymbol{\lambda}, p}^{(q, k)} \cap \operatorname{supp} \boldsymbol{\psi}_{\boldsymbol{\lambda}^{\prime}, p^{\prime}}^{(q, k)} \neq \emptyset$.

For $q=0$, the so extended statement is equal to that of Theorem 3.2. Let us assume that the statement is valid for some $0 \leq q-1 \leq N-1$.

To prove the statement for $q$, it is sufficient to consider $k=\bar{k}$. Let $\varrho$ be a smooth function on $\mathbb{R}^{n}$ such that for some sufficiently small $\varepsilon_{2}>\varepsilon_{1}>0, \varrho \equiv 1$ 
within distance $\varepsilon_{1}$ of the interface $J$ between $\hat{\Omega}_{k_{1}}^{(q-1)}$ and $\hat{\Omega}_{k_{2}}^{(q-1)}$, and vanishes outside distance $\varepsilon_{2}$ of $J$. Writing any function $v$ on $\hat{\Omega}_{\bar{k}}^{(q)}$ as $\varrho v+(1-\varrho) v$ induces a stable splitting of $\stackrel{\circ}{H}^{m}\left(\hat{\Omega}_{\bar{k}}^{(q)}\right) \cap \prod_{\square_{k^{\prime}} \subset \hat{\Omega}_{\bar{k}}^{(q)}} \mathcal{H}_{m, \theta}^{d}\left(\square_{k^{\prime}}\right)$ into two subspaces.

For functions $u$ of type $(1-\varrho) v$, one has $\left.u\right|_{\hat{\Omega}_{k_{2}}^{(q-1)}} \in \stackrel{\circ}{H}^{m}\left(\hat{\Omega}_{k_{2}}^{(q-1)}\right)$ and, assuming $\rho$ to be sufficiently small, $\left\langle\left. u\right|_{\hat{\Omega}_{k_{1}}^{(q-1)}}, \tilde{\boldsymbol{\psi}}_{\boldsymbol{\lambda}, p}^{\left(q-1, k_{1}\right)}\right\rangle_{L_{2}\left(\hat{\Omega}_{k_{1}}^{(q-1)}\right)}=0$ for all $(\boldsymbol{\lambda}, p) \in \boldsymbol{\nabla}_{k_{1}}^{(q-1)}$ with $\eta_{1}^{(q)} \boldsymbol{\psi}_{\boldsymbol{\lambda}, p}^{\left(q-1, k_{1}\right)} \neq E_{1}^{(q)} \boldsymbol{\psi}_{\boldsymbol{\lambda}, p}^{\left(q-1, k_{1}\right)}$. We conclude that for such functions (5.11) is valid when

$$
\nabla_{\bar{k}, M}^{(q)} \supseteq \nabla_{k_{1}, M}^{(q-1)} \cup \nabla_{k_{2}, M}^{(q-1)}
$$

In the remainder of this proof, we consider functions of type $u=\varrho v$, so with support inside some sufficiently small neighborhood of $J$. For $q-1 \leq k \leq N$, we set the biorthogonal projectors

$$
P_{k, M}^{(q-1)}: v \mapsto \sum_{(\boldsymbol{\lambda}, p) \in \nabla_{k, M}^{(q-1)}}\left\langle v, \tilde{\boldsymbol{\psi}}_{\boldsymbol{\lambda}, p}^{(q-1, k)}\right\rangle_{L_{2}\left(\hat{\Omega}_{k}^{(q-1)}\right)} \boldsymbol{\psi}_{\boldsymbol{\lambda}, p}^{(q-1, k)} .
$$

W.l.o.g. we assume $J=\{0\} \times \breve{J}$ and define the (scale-independent) extension $\hat{E}_{1}^{(q)}$ as $E_{1}^{(q)}$ with $G_{1}$ reading as $\hat{G}_{1}$, defined by $\hat{G}_{1} v(-x)=\sum_{l=0}^{L} \gamma_{l} v\left(2^{l} x\right)$ and $\hat{G}_{1} v(x)=v(x)(x \in \mathcal{I})$. So $\hat{G}_{1}$ is the Hestenes extension $\breve{G}_{1}$ without the smooth cut-off function which is not needed here because of the assumption on supp $u$.

It holds that $R_{2}^{(q)}\left(\mathrm{Id}-\hat{E}_{1}^{(q)} R_{1}^{(q)}\right) u \in \stackrel{\circ}{H}^{m}\left(\hat{\Omega}_{k_{2}}^{(q-1)}\right)$ and $R_{1}^{(q)} u \in \stackrel{\circ}{H}^{m}\left(\hat{\Omega}_{k_{1}}^{(q-1)}\right)$. Since $\hat{E}_{1}^{(q)}$ preserves the piecewise weighted Sobolev smoothness of a function supported near the interface, we have

$$
\begin{aligned}
& \sum_{\square_{k^{\prime}} \subset \hat{\Omega}_{k_{2}}^{(q-1)}}\left\|R_{2}^{(q)}\left(\mathrm{Id}-\hat{E}_{1}^{(q)} R_{1}^{(q)}\right) u\right\|_{\mathcal{H}_{m, \theta}^{d}\left(\square_{k^{\prime}}\right)}^{2}+\sum_{\square_{k^{\prime}} \subset \hat{\Omega}_{k_{1}}^{(q-1)}}\left\|R_{1}^{(q)} u\right\|_{\mathcal{H}_{m, \theta}^{d}\left(\square_{k^{\prime}}\right)}^{2} \\
\lesssim & \sum_{\square_{k^{\prime}} \subset \hat{\Omega}_{\bar{k}}^{(q)}}\|u\|_{\mathcal{H}_{m, \theta}^{d}\left(\square_{k^{\prime}}\right)^{2}}^{2}
\end{aligned}
$$

Setting $u_{1}:=P_{k_{1}, M}^{(q-1)} R_{1}^{(q)} u, u_{2}:=P_{k_{2}, M}^{(q-1)} R_{2}^{(q)}\left(\operatorname{Id}-\hat{E}_{1}^{(q)} R_{1}^{(q)}\right) u$, from $\left[\hat{E}_{1}^{(q)} \eta_{2}^{(q)}\right] \in$ $B\left(\stackrel{\circ}{H}^{m}\left(\hat{\Omega}_{k_{1}}^{(q-1)}\right) \times \stackrel{\circ}{H}^{m}\left(\hat{\Omega}_{k_{2}}^{(q-1)}\right), \stackrel{\circ}{H}^{m}\left(\hat{\Omega}_{\bar{k}}^{(q)}\right)\right)$ (see Proposition 4.2), we conclude that

$$
\begin{aligned}
\| u- & \left(\hat{E}_{1}^{(q)} u_{1}+\eta_{2}^{(q)} u_{2}\right) \|_{H^{m}\left(\hat{\Omega}_{\bar{k}}^{(q)}\right)} \\
& \left.=\| \begin{array}{ll}
{\left[\hat{E}_{1}^{(q)}\right.} & \eta_{2}^{(q)}
\end{array}\right]\left(\left[\begin{array}{c}
R_{1}^{(q)} \\
R_{2}^{(q)}\left(\operatorname{Id}-\hat{E}_{1}^{(q)} R_{1}^{(q)}\right)
\end{array}\right] u-\left[\begin{array}{l}
u_{1} \\
u_{2}
\end{array}\right]\right) \|_{H^{m}\left(\hat{\Omega}_{\bar{k}}^{(q)}\right)} \\
& \lesssim \sqrt{\left\|R_{1}^{(q)} u-u_{1}\right\|_{H^{m}\left(\hat{\Omega}_{k_{1}}^{(q-1)}\right)}^{2}+\left\|R_{2}^{(q)}\left(\mathrm{Id}-\hat{E}_{1}^{(q)} R_{1}^{(q)}\right) u-u_{2}\right\|_{H^{m}\left(\hat{\Omega}_{k_{2}}^{(q-1)}\right)}^{2}} \\
& \lesssim M^{-(d-m)} \sqrt{\sum_{\square_{k^{\prime}} \subset \hat{\Omega}_{\bar{k}}^{(q)}}\|u\|_{\mathcal{H}_{m, \theta}^{d}\left(\square_{k}^{\prime}\right)}^{2}},
\end{aligned}
$$

the last inequality by the induction hypothesis and (5.12).

Next, we write

$$
u-\left(E_{1}^{(q)} u_{1}+\eta_{2}^{(q)} u_{2}\right)=u-\left(\hat{E}_{1}^{(q)} u_{1}+\eta_{2}^{(q)} u_{2}\right)+\left(\hat{E}_{1}^{(q)}-E_{1}^{(q)}\right) u_{1} .
$$


By construction of $G_{1}$ from $\breve{G}_{1}$, we have that $\left(\operatorname{Id}-\eta_{2}^{(q)} R_{2}^{(q)}\right)\left(\hat{E}_{1}^{(q)}-E_{1}^{(q)}\right) u_{1}=0$, and $R_{2}^{(q)}\left(\hat{E}_{1}^{(q)}-E_{1}^{(q)}\right) u_{1} \in \stackrel{\circ}{H}^{m}\left(\hat{\Omega}_{k_{2}}^{(q-1)}\right)$, and so

$$
\left(\hat{E}_{1}^{(q)}-E_{1}^{(q)}\right) u_{1}=\sum_{(\hat{\boldsymbol{\lambda}}, \hat{p}) \in \nabla_{k_{2}}^{(q-1)}}\left\langle R_{2}^{(q)}\left(\hat{E}_{1}^{(q)}-E_{1}^{(q)}\right) u_{1}, \tilde{\boldsymbol{\psi}}_{\hat{\boldsymbol{\lambda}}, \hat{p}}^{\left(q-1, k_{2}\right)}\right\rangle_{L_{2}\left(\hat{\Omega}_{k_{2}}^{(q-1)}\right)} \eta_{2}^{(q)} \boldsymbol{\psi}_{\hat{\boldsymbol{\lambda}}, \hat{p}}^{\left(q-1, k_{2}\right)} .
$$

We set

$$
\begin{array}{r}
\hat{\nabla}_{k_{2}, M}^{(q-1)}:=\left\{(\hat{\boldsymbol{\lambda}}, \hat{p}) \in \nabla_{k_{2}}^{(q-1)}:\left\langle R_{2}^{(q)}\left(\hat{E}_{1}^{(q)}-E_{1}^{(q)}\right) u_{1}, \tilde{\boldsymbol{\psi}}_{\hat{\boldsymbol{\lambda}}, \hat{p}}^{\left(q-1, k_{2}\right)}\right\rangle_{L_{2}\left(\hat{\Omega}_{k_{2}}^{(q-1)}\right)} \neq 0,\right. \\
\text { for some } \left.u_{1} \in \Im P_{k_{1}, M}^{(q-1)}\right\} .
\end{array}
$$

Below we will show that, even after a possible enlargement to ensure the multiple tree property, it holds that $\# \hat{\nabla}_{k_{2}, M}^{(q-1)} \lesssim \# \nabla_{k_{1}, M}^{(q-1)}$. Defining $\nabla_{\bar{k}, M}^{(q)}:=\nabla_{k_{1}, M}^{(q-1)} \cup$ $\nabla_{k_{2}, M}^{(q-1)} \cup \hat{\nabla}_{k_{2}, M}^{(q-1)}$, the proof is completed.

If $(\hat{\boldsymbol{\lambda}}, \hat{p}) \in \hat{\boldsymbol{\nabla}}_{k_{2}, M}^{(q-1)}$, then $\left\langle R_{2}^{(q)} \hat{E}_{1}^{(q)} \boldsymbol{\psi}_{\boldsymbol{\lambda}, p}^{\left(q-1, k_{1}\right)}, \tilde{\boldsymbol{\psi}}_{\hat{\boldsymbol{\lambda}}, \hat{p}}^{\left(q-1, k_{2}\right)}\right\rangle_{L_{2}\left(\hat{\Omega}_{k_{2}}^{(q-1)}\right)} \neq 0$ for some $(\boldsymbol{\lambda}, p) \in \nabla_{k_{1}, M}^{(q-1)}$ with $R_{2}^{(q)} \hat{E}_{1}^{(q)} \boldsymbol{\psi}_{\boldsymbol{\lambda}, p}^{\left(q-1, k_{1}\right)} \in \stackrel{\circ}{H}^{m}\left(\hat{\Omega}_{k_{2}}^{(q-1)}\right)$. Using $Z_{|\boldsymbol{\lambda}|}\left(\hat{\Omega}_{k_{1}}^{(q-1)}\right) \subset$ $V_{|\boldsymbol{\lambda}|+M_{q-1} \mathbf{e}}\left(\hat{\Omega}_{k_{1}}^{(q-1)}\right)$ and the assumptions on the extension, we have

$$
R_{2}^{(q)} \hat{E}_{1}^{(q)} \boldsymbol{\psi}_{\boldsymbol{\lambda}, p}^{\left(q-1, k_{1}\right)} \in V_{|\boldsymbol{\lambda}|+\left(M_{q-1}+L\right) \mathbf{e}}\left(\hat{\Omega}_{k_{2}}^{(q-1)}\right) \subset Z_{|\boldsymbol{\lambda}|+\left(M_{q-1}+m_{q-1}+L\right) \mathbf{e}}\left(\hat{\Omega}_{k_{2}}^{(q-1)}\right),
$$

so $|\hat{\boldsymbol{\lambda}}| \leq|\boldsymbol{\lambda}|+\left(M_{q-1}+m_{q-1}+L\right)$ e. Here we applied both inclusions from Proposition $5.4(3)$.

Thanks to the multiple tree property of $\nabla_{k_{1}, M}^{(q-1)}$, there exists a $\left(\boldsymbol{\lambda}^{\prime}, p^{\prime}\right) \in \boldsymbol{\nabla}_{k_{1}, M}^{(q-1)}$ with $\left|\boldsymbol{\lambda}^{\prime}\right|_{i}=\min \left(|\hat{\boldsymbol{\lambda}}|_{i},|\boldsymbol{\lambda}|_{i}\right)(1 \leq i \leq n)$ and $\operatorname{supp} \boldsymbol{\psi}_{\boldsymbol{\lambda}, p}^{\left(q-1, k_{1}\right)} \cap \operatorname{supp} \boldsymbol{\psi}_{\boldsymbol{\lambda}^{\prime}, p^{\prime}}^{\left(q-1, k_{1}\right)} \neq \emptyset$. Note that because of $|\hat{\boldsymbol{\lambda}}| \leq|\boldsymbol{\lambda}|+\left(M_{q-1}+m_{q-1}+L\right)$ e, we have $\left|\boldsymbol{\lambda}^{\prime}\right| \leq|\hat{\boldsymbol{\lambda}}| \leq$ $\left|\boldsymbol{\lambda}^{\prime}\right|+\left(M_{q-1}+m_{q-1}+L\right) \mathbf{e}$.

The "localness" of $\boldsymbol{\Psi}_{k_{1}}^{(q-1)}$ as given by Proposition 5.4(1), the assumptions on the extension, and the "locally finiteness" of $\tilde{\boldsymbol{\Psi}}_{k_{2}}^{(q-1)}$ as given by Proposition 5.4(2) show that the number of $(\hat{\boldsymbol{\lambda}}, \hat{p}) \in \hat{\boldsymbol{\nabla}}_{k_{2}, M}^{(q-1)}$ with $\left\langle R_{2}^{(q)} \hat{E}_{1}^{(q)} \boldsymbol{\psi}_{\boldsymbol{\lambda}, p}^{\left(q-1, k_{1}\right)}, \tilde{\boldsymbol{\psi}}_{\hat{\boldsymbol{\lambda}}, \hat{p}}^{\left(q-1, k_{2}\right)}\right\rangle_{L_{2}\left(\hat{\Omega}_{k_{2}}^{(q-1)}\right)} \neq 0$ on the same level $|\hat{\boldsymbol{\lambda}}| \leq|\boldsymbol{\lambda}|+\left(M_{q-1}+m_{q-1}+L\right)$ e is uniformly bounded. With this, we conclude that with the above mapping $(\hat{\boldsymbol{\lambda}}, \hat{p}) \mapsto\left(\boldsymbol{\lambda}^{\prime}, p^{\prime}\right)$, an at most uniformly bounded number of $(\hat{\boldsymbol{\lambda}}, \hat{p}) \in \hat{\boldsymbol{\nabla}}_{k_{2}, M}^{(q-1)}$ is mapped onto any $\left(\boldsymbol{\lambda}^{\prime}, p^{\prime}\right) \in \boldsymbol{\nabla}_{k_{1}, M}^{(q-1)}$, so that $\# \hat{\boldsymbol{\nabla}}_{k_{2}, M}^{(q-1)} \lesssim \# \boldsymbol{\nabla}_{k_{1}, M}^{(q-1)}$.

Finally, to bound $\# \hat{\nabla}_{k_{2}, M}^{(q-1)}$, we only used that for $(\hat{\boldsymbol{\lambda}}, \hat{p}) \in \hat{\boldsymbol{\nabla}}_{k_{2}, M}^{(q-1)}$ there exists a $(\boldsymbol{\lambda}, p) \in \boldsymbol{\nabla}_{k_{1}, M}^{(q-1)}$ with $\operatorname{supp} R_{2}^{(q)} \hat{E}_{1}^{(q)} \boldsymbol{\psi}_{\boldsymbol{\lambda}, p}^{\left(q-1, k_{1}\right)} \cap \operatorname{supp} \tilde{\boldsymbol{\psi}}_{\hat{\boldsymbol{\lambda}}, \hat{p}}^{\left(q-1, k_{1}\right)} \neq \emptyset$ and $|\hat{\boldsymbol{\lambda}}| \leq$ $|\boldsymbol{\lambda}|+\left(M_{q-1}+m_{q-1}+L\right)$ e. The same proof would have applied with the condition about the non-empty intersection of the supports reading as the condition that supp $R_{2}^{(q)} \hat{E}_{1}^{(q)} \boldsymbol{\psi}_{\boldsymbol{\lambda}, p}^{\left(q-1, k_{1}\right)}$ has non-empty intersection with some hyper-rectangle, containing $\operatorname{supp} \tilde{\boldsymbol{\psi}}_{\hat{\boldsymbol{\lambda}}, \hat{p}}^{\left(q-1, k_{1}\right)}$, that is aligned with the Cartesian coordinates with sides of lengths of order $2^{-|\hat{\boldsymbol{\lambda}}|_{1}}, \ldots, 2^{-|\hat{\boldsymbol{\lambda}}|_{n}}$. In view of this, if $\hat{\boldsymbol{\nabla}}_{k_{2}, M}^{(q-1)}$ does not already have the multiple tree property, then it can be enlarged to have this property while retaining $\# \hat{\nabla}_{k_{2}, M}^{(q-1)} \lesssim \# \boldsymbol{\nabla}_{k_{1}, M}^{(q-1)}$. 


\section{Regularity}

We study the issue whether we may expect (5.1) for $u$ being the solution of an elliptic boundary value problem of order $2 m=2$.

6.1. Two-dimensional case. Let $\Omega$ be a polygonal domain. This means that its boundary is the union of a finite number of line segments, knowns as edges, with ends known as corners. It is not assumed that $\Omega$ is a Lipschitz domain, so it may contain cracks. We denote with $\mathcal{E}$ the set of edges, with $\mathcal{C}$ the set of corners, and set for $\mathbf{c} \in \mathcal{C}$,

$$
r_{\mathbf{c}}(\mathbf{x}):=\operatorname{dist}(\mathbf{x}, \mathbf{c}) .
$$

Following CDN10, for $m \in \mathbb{N}_{0}$, we define the (non-homogeneous) weighted Sobolev space $J_{\beta}^{m}(\Omega)$ as the set of $u \in L_{2}^{\text {loc }}(\Omega)$ that have a finite squared norm

$$
\|v\|_{J_{\beta}^{m}(\Omega)}^{2}:=\sum_{k=0}^{m} \sum_{|\alpha|=k}\left\|\left\{\prod_{\mathbf{c} \in \mathcal{C}} r_{\mathbf{c}}^{\beta+m}\right\} \partial^{\alpha} v\right\|_{L_{2}(\Omega)}^{2} .
$$

(in CDN10 the generalization is considered of $\beta$ being possibly dependent on $\mathbf{c}$ ).

Let $A$ be a constant, real, symmetric and positive definite $2 \times 2$ matrix. Let $\mathcal{E}_{D} \subset \mathcal{E}$, and

$$
V(\Omega):= \begin{cases}\left\{v \in H^{1}(\Omega):\left.v\right|_{\mathbf{e}}=0 \forall \mathbf{e} \in \mathcal{E}_{D}\right\} & \text { when } \mathcal{E}_{D} \neq \emptyset \\ \left\{v \in H^{1}(\Omega): \int_{\Omega} v d x=0\right\} & \text { otherwise. }\end{cases}
$$

Given $g \in V(\Omega)^{\prime}$, let $u \in V(\Omega)$ denote the solution of

$$
\int_{\Omega} A \nabla u \cdot \nabla v d x=g(v) \quad(v \in V(\Omega)) .
$$

Theorem 6.1. For $m \in \mathbb{N}_{0}$, there exists a $b^{*} \in(0, m+2]$ such that for any $b \in\left[0, b^{*}\right)$, the mapping $g \mapsto u \in B\left(J_{-b+1}^{m}(\Omega), J_{-b-1}^{m+2}(\Omega)\right)$.

The proof follows from [CDN10, formula (6.7)]. As stated in CDN10, Example 6.7], for $m$ sufficiently large, $b^{*}>\frac{1}{4}$.

We refer to [CDN10, Sect. 7] for generalizations of Theorem 6.1 to differential operators with variable coefficients and/or lower order terms.

Concerning the smoothness condition on the right-hand side $g$, note that for $b \leq m+1$,

$$
H^{m}(\Omega) \hookrightarrow J_{-b+1}^{m}(\Omega) .
$$

Let us now consider the situation that $\Omega=\bigcup_{i=1}^{K} \Omega_{i}$ is an essentially disjoint subdivision into subdomains, where $\Omega_{i}=\kappa_{i}(\square)$ with $\kappa_{i}$ being a regular parametrization. Let $R_{i}$ denote the restriction of functions on $\Omega$ to $\Omega_{i}$.

Proposition 6.2. If $\kappa_{i} \in C^{m+2}(\bar{\square})$ and $b \leq m+1$, then

$$
\kappa_{i}^{*} R_{i} \in B\left(J_{-b-1}^{m+2}(\Omega), J_{-b-1}^{m+2}(\square)\right) .
$$

Proof. This follows from the smoothness of $\kappa_{i}$, and from the fact that $\left.\kappa_{i}^{*} u\right|_{\Omega_{i}}$ localized near corners of $\square$ that do not correspond to corners of $\Omega$ is a function in $H^{m+2} \hookrightarrow J_{-b-1}^{m+2}$, the latter by $-b-1+m+2 \geq 0$.

The following proposition demonstrates (5.1). 
Proposition 6.3. For $d \in \mathbb{N}_{0}, \theta \geq \max (1, d-b / 2)$, it holds that

$$
J_{-b-1}^{2 d}(\square) \hookrightarrow \mathcal{H}_{\theta-1}^{d}(\mathcal{I}) \otimes \mathcal{H}_{\theta}^{d}(\mathcal{I}) \cap \mathcal{H}_{\theta}^{d}(\mathcal{I}) \otimes \mathcal{H}_{\theta-1}^{d}(\mathcal{I})=\mathcal{H}_{1, \theta}^{d}(\square) .
$$

Proof. This follows from $\max \left(x^{\theta-1} y^{\theta}, x^{\theta} y^{\theta-1}\right) \leq r_{\mathbf{0}}^{2 \theta-1} \leq r_{\mathbf{0}}^{2 d-b-1}$ when $r_{\mathbf{0}} \in[0,1]$.

6.2. Three-dimensional case. As in the previous section we follow CDN10] closely. Let $\Omega$ be a polyhedral domain. This means that its boundary is the union of a finite number of polygons, known as the faces; the segments forming their boundaries are the edges, and the ends of the edges are the corners. It is not assumed that $\Omega$ is a Lipschitz domain, so it may contain crack surfaces. We denote with $\mathcal{F}$, $\mathcal{E}$, and $\mathcal{C}$ the set of faces, edges, and corners, respectively, and set for $\mathbf{e} \in \mathcal{E}$ and $\mathbf{c} \in \mathcal{C}$

$$
r_{\mathbf{e}}(\mathbf{x}):=\operatorname{dist}(\mathbf{x}, \mathbf{e}), r_{\mathbf{c}}(\mathbf{x}):=\operatorname{dist}(\mathbf{x}, \mathbf{c}), r_{\mathcal{C}}(\mathbf{x}):=\min _{\mathbf{c} \in \mathcal{C}} r_{\mathbf{c}}(\mathbf{x}), r_{\mathcal{E}}(\mathbf{x}):=\min _{\mathbf{e} \in \mathcal{E}} r_{\mathbf{e}}(\mathbf{x}) .
$$

There exists an $\varepsilon>0$ small enough such that if we set

$$
\begin{aligned}
\Omega_{\mathbf{e}} & :=\left\{\mathbf{x} \in \Omega: r_{\mathbf{e}}(\mathbf{x})<\varepsilon, r_{\tilde{\mathbf{e}}}(\mathbf{x})>r_{\mathbf{e}}(\mathbf{x})(\mathbf{e} \neq \tilde{\mathbf{e}} \in \mathcal{E}), \text { and } r_{\mathcal{C}}(\mathbf{x})>\frac{\varepsilon}{2}\right\}, \\
\Omega_{\mathbf{c}} & :=\left\{\mathbf{x} \in \Omega: r_{\mathbf{c}}(\mathbf{x})<\varepsilon \text { and } r_{\mathcal{E}}(\mathbf{x})>\frac{\varepsilon}{2} r_{\mathbf{c}}(\mathbf{x})\right\}, \\
\Omega_{\mathbf{c e}} & :=\left\{\mathbf{x} \in \Omega: r_{\mathbf{c}}(\mathbf{x})<\varepsilon \text { and } r_{\mathbf{e}}(\mathbf{x})<\varepsilon r_{\mathbf{c}}(\mathbf{x})\right\}, \\
\Omega_{I} & :=\left\{\mathbf{x} \in \Omega: r_{\mathcal{E}}(\mathbf{x})>\frac{\varepsilon}{2}\right\},
\end{aligned}
$$

we have the following properties:

$$
\bar{\Omega}_{\mathbf{e}} \cap \bar{\Omega}_{\mathbf{e}^{\prime}}=\emptyset, \quad \bar{\Omega}_{\mathbf{c e}} \cap \bar{\Omega}_{\mathbf{c e}^{\prime}}=\{\mathbf{c}\} \quad\left(\mathbf{e} \neq \mathbf{e}^{\prime} \in \mathcal{E}, \mathbf{c} \in \mathcal{C}\right),
$$

$\overline{B(\mathbf{c} ; \varepsilon)} \cap \overline{B\left(\mathbf{c}^{\prime} ; \varepsilon\right)}=\emptyset \quad\left(\mathbf{c} \neq \mathbf{c}^{\prime} \in \mathcal{C}\right), \quad \Omega=\Omega_{I} \cup_{\{\mathbf{c} \in \mathcal{C}\}} \Omega_{\mathbf{c}} \cup_{\{\mathbf{e} \in \mathcal{E}\}} \Omega_{\mathbf{e}} \cup_{\{\mathbf{c} \in \mathcal{C}, \mathbf{e} \in \mathcal{E}\}} \Omega_{\mathbf{c e}}$.

In a neighborhood of any edge $\mathbf{e} \in \mathcal{E}$, we will take partial derivatives in an orthogonal coordinate system with one of the coordinate directions being parallel to $e$. For a multi-index $\alpha$ in that coordinate system, $\left|\alpha_{\perp}\right|$ will denote the sum of the coordinates in the directions perpendicular to $e$, and $\left|\alpha_{\|}\right|:=|\alpha|-\left|\alpha_{\perp}\right|$.

For $m \in \mathbb{N}_{0}, \beta>-m$, and $\mathcal{E}_{0} \subset \mathcal{E}$, we define the anisotropic weighted Sobolev space

$$
\begin{aligned}
N_{\beta}^{m}\left(\Omega, \mathcal{C}, \mathcal{E}_{0}\right):=\left\{u \in L_{2}^{\mathrm{loc}}(\Omega): \forall \alpha,|\alpha| \leq m, \partial^{\alpha} u\right. & \in L_{2}\left(\Omega_{I}\right) \\
r_{\mathbf{c}}(\mathbf{x})^{\beta+|\alpha|} \partial^{\alpha} u & \in L_{2}\left(\Omega_{\mathbf{c}}\right) \forall \mathbf{c} \in \mathcal{C}, \\
r_{\mathbf{e}}(\mathbf{x})^{\beta+\left|\alpha_{\perp}\right|} \partial^{\alpha} u & \in L_{2}\left(\Omega_{\mathbf{e}}\right) \forall \mathbf{e} \in \mathcal{E}_{0}, \\
r_{\mathbf{c}}(\mathbf{x})^{\beta+|\alpha|}\left(r_{\mathbf{e}}(\mathbf{x}) / r_{\mathbf{c}}(\mathbf{x})\right)^{\beta+\left|\alpha_{\perp}\right|} \partial^{\alpha} u & \in L_{2}\left(\Omega_{\mathbf{c e}}\right) \forall \mathbf{c} \in \mathcal{C}, \mathbf{e} \in \mathcal{E}_{0} \\
r_{\mathbf{e}}(\mathbf{x})^{\max \left(\beta+\left|\alpha_{\perp}\right|, 0\right)} \partial^{\alpha} u & \in L_{2}\left(\Omega_{\mathbf{e}}\right) \forall \mathbf{e} \in \mathcal{E} \backslash \mathcal{E}_{0}, \\
r_{\mathbf{c}}(\mathbf{x})^{\beta+|\alpha|}\left(r_{\mathbf{e}}(\mathbf{x}) / r_{\mathbf{c}}(\mathbf{x})\right)^{\max \left(\beta+\left|\alpha_{\perp}\right|, 0\right)} \partial^{\alpha} u & \left.\in L_{2}\left(\Omega_{\mathbf{c e}}\right) \forall \mathbf{c} \in \mathcal{C}, \mathbf{e} \in \mathcal{E} \backslash \mathcal{E}_{0}\right\},
\end{aligned}
$$

with squared norm being the sum over $|\alpha| \leq m$ of the squared $L_{2}$-norms over $\Omega_{I}$, $\Omega_{\mathbf{c}}, \Omega_{\mathbf{e}}, \Omega_{\mathbf{c e}}$, and $\mathbf{c} \in \mathcal{C}, \mathbf{e} \in \mathcal{E}$, respectively. (As in the two-dimensional case, this definition can be generalized to $\beta$ being possibly dependent on $\mathbf{c}$ and $\mathbf{e})$.

The definition of $N_{\beta}^{m}\left(\Omega, \mathcal{C}, \mathcal{E}_{0}\right)$ is a special case of a definition of $N_{\beta}^{m}\left(\Omega, \mathcal{C}_{0}, \mathcal{E}_{0}\right)$ from $\left[\mathrm{CDN10}\right.$ for general $\mathcal{C}_{0} \subseteq \mathcal{C}$. In particular, the definition of the (fully) nonhomogeneous anisotropic weighted Sobolev space $N_{\beta}^{m}(\Omega):=N_{\beta}^{m}(\Omega, \emptyset, \emptyset)$ is obtained 
from (6.2) by taking $\mathcal{E}_{0}=\emptyset$, and by replacing $r_{\mathbf{c}}(\mathbf{x})^{\beta+|\alpha|}$ by $r_{\mathbf{c}}(\mathbf{x})^{\max (\beta+|\alpha|, 0)}$ on all three occurrences. Obviously,

$$
N_{\beta}^{m}\left(\Omega, \mathcal{C}, \mathcal{E}_{0}\right) \hookrightarrow N_{\beta}^{m}(\Omega) .
$$

Let $A$ be a constant, real, symmetric and positive definite $3 \times 3$ matrix. Let $\mathcal{F}_{D} \subset \mathcal{F}$, and

$$
V(\Omega):= \begin{cases}\left\{v \in H^{1}(\Omega):\left.v\right|_{\mathbf{f}}=0 \forall \mathbf{f} \in \mathcal{F}_{D}\right\} & \text { when } \mathcal{F}_{D} \neq \emptyset \\ \left\{v \in H^{1}(\Omega): \int_{\Omega} v d x=0\right\} & \text { otherwise. }\end{cases}
$$

Given $g \in V(\Omega)^{\prime}$, let $u \in V(\Omega)$ denote the solution of

$$
\int_{\Omega} A \nabla u \cdot \nabla v d x=g(v) \quad(v \in V(\Omega)) .
$$

Theorem 6.4. Let $\mathcal{E}_{0}$ be the set of all $\mathbf{e} \in \mathcal{E}$ that are an edge of an $\mathbf{f} \in \mathcal{F}_{D}$. There exists $a b^{*} \in(0,1]$ such that for $m \in \mathbb{N}, m>1$, and for any $b \in\left[0, b^{*}\right)$, the mapping $g \mapsto u \in B\left(N_{1-b}^{m}\left(\Omega, \mathcal{C}, \mathcal{E}_{0}\right), N_{-1-b}^{m}\left(\Omega, \mathcal{C}, \mathcal{E}_{0}\right)\right)$.

Indeed, with the isotropic weighted Sobolev spaces $J_{\beta}^{m}(\Omega)$ as defined in CDN10. Def. 5.9] (where we consider the value of $\beta$ to be independent of the edges and corners), MR03, Th. 7.1] shows that $g \mapsto u \in B\left(J_{1-b}^{0}(\Omega), J_{1-b}^{2}(\Omega)\right)$, and thus that $g \mapsto u \in B\left(J_{1-b}^{0}(\Omega), J_{1-b}^{1}(\Omega)\right)$. Using that $\left.N_{1-b}^{m}\left(\Omega, \mathcal{C}, \mathcal{E}_{0}\right) \hookrightarrow J_{1-b}^{0}(\Omega)\right)$, we conclude the statement of the theorem from the anisotropic regularity shift theorem CDN10, (5.25)(a)]. Here we used that the Assumptions 5.5 and 5.13 from [CDN10] for $\mathbf{e} \in \mathcal{E}_{0}$ or $\mathbf{e} \in \mathcal{E} \backslash \mathcal{E}_{0}$, respectively, are satisfied by an application of MR03, Th. $7.2]$.

Concerning the smoothness condition on the right-hand side $g$, note that for $b \leq 1$,

$$
H^{m}(\Omega) \hookrightarrow N_{1-b}^{m}\left(\Omega, \mathcal{C}, \mathcal{E}_{0}\right) .
$$

The fact, as proven in Theorem 6.4, that for sufficiently smooth right-hand side, the tangential derivatives of sufficiently high order along the edges of $\Omega$ of the solution of (6.4) are in the (unweighted) $L_{2}(\Omega)$ space, is essential for our goal of proving approximation rates with piecewise tensor product approximation as for one-dimensional problems.

Let us consider the situation that $\Omega=\bigcup_{i=1}^{K} \Omega_{i}$ is an essentially disjoint conforming subdivision into hexahedra that are images of $\square$ under trilinear diffeomorphisms $\kappa_{i}$, with $\inf _{\mathbf{x} \in \square}\left|D \kappa_{i}(\mathbf{x})\right|>0$.

Aiming at deriving a three-dimensional analogue of Proposition 6.2, care has to be taken that for $\kappa_{i}^{*} R_{i} u$ to be in $N_{-1-b}^{m}(\square)$, its tangential derivatives along an edge up to order $m$ have to be in $L_{2}(\square)$. Therefore, we have to ensure that if an edge of $\square$ is mapped onto the boundary of $\Omega$, then lines parallel to this edge are (smoothly) mapped onto lines parallel to the boundary of $\Omega$.

Proposition 6.5. For any $i$ let $\kappa_{i}$ be such that if it maps an edge $\mathbf{e}$ of $\square$ to an edge of $\Omega$, then it maps all three edges that are parallel to e to edges that are parallel to $\kappa_{i}(\mathbf{e})$. Then

$$
\kappa_{i}^{*} R_{i} \in B\left(N_{-1-b}^{m}(\Omega), N_{-1-b}^{m}(\square)\right) .
$$

Proof. What has to be shown is that if an edge e of $\square$ is mapped to the boundary of $\Omega$, then the tangential derivatives along e of $u \circ \kappa_{i}$ up to order $m$ are a smooth functions of the tangential derivatives of $u$ along $\kappa_{i}(\mathbf{e})$ up to order $m$. 


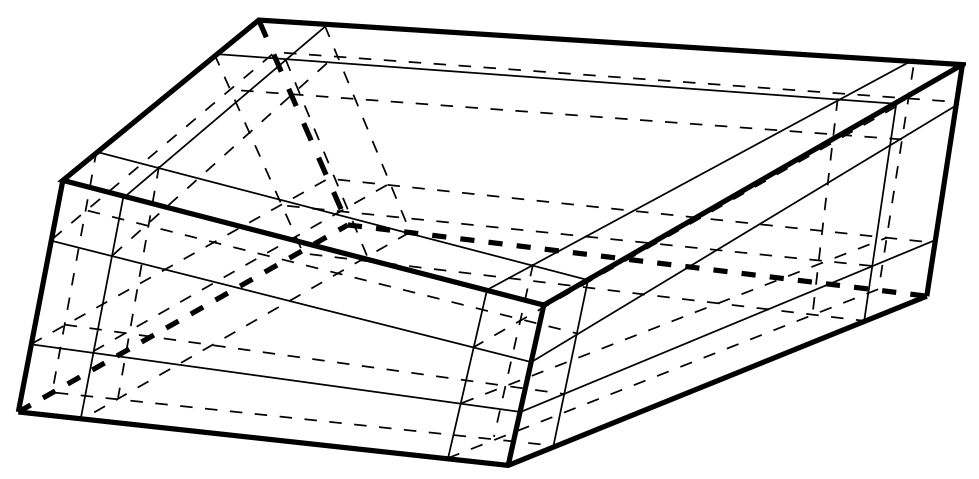

Figure 5. Hexahedron cut into $3^{3}$ subhexahedra.

W.l.o.g., let $\mathbf{e}$ be one of the edges $\mathbf{e}^{(1)}, \ldots, \mathbf{e}^{(4)}$ that are parallel to the first unit vector. The vector $\partial_{1} \kappa_{i}(\mathbf{x})$ is a bilinear function of $x_{2}$ and $x_{3}$, and so, in particular, constant on each of the $\mathbf{e}^{(j)}$. These constant vectors are the differences of the endpoints of $\kappa_{i}\left(\mathbf{e}^{(j)}\right)$, and so, by assumption, multiples of $\left.\partial_{1} \kappa_{i}\right|_{\mathbf{e}}$. We conclude that $\partial_{1} \kappa_{i}(\mathbf{x})$ is a multiple of a bilinear scalar function and $\left.\partial_{1} \kappa_{i}\right|_{\mathbf{e}}$.

Next we will show that the condition on the parametrizations imposed in Proposition 6.5 can always be satisfied by making some refinement of the initial conforming subdivision into hexahedra: Let us cut each hexahedron in the partition along 6 planes parallel to the 6 faces of the hexahedron on distance $\zeta>0$; see Figure 5. When $\zeta$ is small enough, then the planes parallel to opposite faces of the hexahedron do not intersect inside the hexahedron, and we obtain a subdivision of the hexahedron in $3^{3}$ hexahedra. Eight of these hexahedra share a corner with the original hexahedron and so have three edges on edges of this hexahedron, and so possibly three edges on edges of $\Omega$. These hexahedra are parallelepipeds and so satisfy the condition from Proposition 6.5. Twelve other hexahedra have one edge on an edge of the original hexahedron, and so possibly on an edge of $\Omega$. For each of these hexahedra, the edges opposite to this specific edge are parallel to this edge and so satisfy the condition from Proposition 6.5. The remaining seven hexahedra have no edges on edges of the original hexahedron, and thus no edge on an edge of $\Omega$. Six of them have a face on a face of the original hexahedron, whereas the boundary of the remaining "interior" hexahedron has empty intersection with the boundary of the original hexahedron.

The above subdivision of a hexahedron induces a subdivision of each of its faces into $3^{2}$ quadrilaterals; 4 parallelograms at the corners, 4 trapezoids at the edges, and one interior quadrilateral. Conversely, such a subdivision of 3 non-opposite faces of the hexahedron, where the interior quadrilaterals are sufficiently large, determines uniquely the subdivision of the hexahedron into $3^{3}$ subhexahedra by making cuts along planes parallel to the faces. So if we start with a subdivision of one hexahedron and use the resulting subdivision of its faces to induce subdivisions of its neighbors, then by choosing $\zeta$ small enough we obtain a refinement of the original conforming decomposition into hexahedra to a conforming decomposition into hexahedra that satisfy the conditions needed for Proposition 6.5.

What is left to show is whether the hexahedra in the refined subdivision are images of $\square$ under trilinear diffeomorphisms $\kappa_{i}$, with $\inf _{\mathbf{x} \in \square}\left|D \kappa_{i}(\mathbf{x})\right|>0$. When 
the aforementioned parameter $\zeta$ tends to zero, the interior hexahedron converges to the hexahedron in the original decomposition, which was assumed to have this property. So for $\zeta$ small enough, the interior hexahedra have this property.

The other hexahedra in the refined subdivision have at least two parallel faces, and so are instances of a prismatoid. Let us consider such a hexahedron with its parallel faces, being convex quadrilaterals, on the planes $z=0$ and $z=1$. Let $q_{1}, q_{2}:(0,1)^{2} \rightarrow \mathbb{R}^{2}$ be bilinear parametrizations of the bottom and top face with $\inf _{(x, y) \in(0,1)^{2}}\left|D q_{i}(x, y)\right|>0$, and such that the images of each corner of $(0,1)^{2}$ under $q_{1}$ and $q_{2}$ are connected by an edge in the hexahedron. Then a trilinear parametrization $\square \rightarrow \mathbb{R}^{3}$ is given by

$$
\kappa(x, y, z)=(1-z) q_{1}(x, y)+z q_{2}(x, y)
$$

and so $\inf _{(x, y, z) \in \square}|D \kappa(x, y, z)|=\inf _{(x, y, z) \in \square}(1-z)\left|D q_{1}(x, y)\right|+z\left|D q_{2}(x, y)\right|>0$.

The following proposition demonstrates (5.1).

Proposition 6.6. For $d \in \mathbb{N}_{0}, \theta \geq \max \left(1, d-\frac{b}{3}\right)$ where $b>0$, it holds that

$$
N_{-1-b}^{3 d}(\square) \hookrightarrow \mathcal{H}_{1, \theta}^{d}(\square) .
$$

Proof. It is sufficient to show continuity of the embedding of the spaces restricted to $\Omega_{\mathbf{c}}, \Omega_{\mathbf{e}}$, and $\Omega_{\mathbf{c e}}$ intersected with $\left(0, \frac{1}{2}\right)^{3}$, where $\mathbf{c}=(0,0,0)$ and $\mathbf{e}=\mathbf{e}_{1}$.

For $\|\alpha\|_{\infty} \leq d$, the conditions on $\theta$ show that on $\Omega_{\mathbf{c}} \cap\left(0, \frac{1}{2}\right)^{3}$,

$$
\max \left(x^{\theta-1} y^{\theta} z^{\theta}, x^{\theta} y^{\theta-1} z^{\theta}, x^{\theta} y^{\theta} z^{\theta-1}\right) \leq r_{\mathbf{c}}(\mathbf{x})^{3 \theta-1} \leq r_{\mathbf{c}}(\mathbf{x})^{\max (-1-b+|\alpha|, 0)},
$$

and on $\Omega_{\mathbf{e}} \cap\left(0, \frac{1}{2}\right)^{3}$,

$$
\max \left(y^{\theta} z^{\theta}, y^{\theta-1} z^{\theta}, y^{\theta} z^{\theta-1}\right) \leq r_{\mathbf{e}}(\mathbf{x})^{2 \theta-1} \leq r_{\mathbf{e}}(\mathbf{x})^{\max \left(-1-b+\alpha_{2}+\alpha_{3}, 0\right)} .
$$

On $\Omega_{\mathbf{c e}} \cap\left(0, \frac{1}{2}\right)^{3}$, we have

$$
\max \left(x^{\theta-1} y^{\theta} z^{\theta}, x^{\theta} y^{\theta-1} z^{\theta}, x^{\theta} y^{\theta} z^{\theta-1}\right) \leq r_{\mathbf{c}}(\mathbf{x})^{\theta} r_{\mathbf{e}}(\mathbf{x})^{2 \theta-1} .
$$

To show that this right-hand side can be bounded on

$$
r_{\mathbf{c}}(\mathbf{x})^{\max (-1-b+|\alpha|, 0)-\max \left(-1-b+\alpha_{2}+\alpha_{3}, 0\right)} r_{\mathbf{e}}(\mathbf{x})^{\max \left(-1-b+\alpha_{2}+\alpha_{3}, 0\right)}
$$

we distinguish between 3 cases: For $-1-b+|\alpha| \leq 0$, this results from $\theta \geq 0$ and $2 \theta-1 \geq 0$. For $-1-b+|\alpha| \geq 0 \geq-1-b+\alpha_{2}+\alpha_{3}$, we have $r_{\mathbf{c}}(\mathbf{x})^{\theta} r_{\mathbf{e}}(\mathbf{x})^{2 \theta-1} \leq$ $r_{\mathbf{c}}(\mathbf{x})^{3 \theta-1} \leq r_{\mathbf{c}}(\mathbf{x})^{-1-b+|\alpha|}$ by $\theta \geq \bar{d}-\frac{b}{3}$. For $-1-b+\alpha_{2}+\alpha_{3} \geq 0, r_{\mathbf{c}}(\mathbf{x})^{\theta} r_{\mathbf{e}}(\mathbf{x})^{2 \theta-1} \leq$ $r_{\mathbf{c}}(\mathbf{x})^{\theta+\frac{b}{3}} r_{\mathbf{e}}(\mathbf{x})^{2 \theta-1-\frac{b}{3}} \leq r_{\mathbf{c}}(\mathbf{x})^{\alpha_{1}} r_{\mathbf{e}}(\mathbf{x})^{-1-b+\alpha_{2}+\alpha_{3}}$ by $\theta \geq d-\frac{b}{3}$.

\section{NuMERICAL RESULTS}

As the univariate building block of the piecewise tensor product wavelet construction, we apply the $C^{1}$, piecewise quartic (so $d=5$ ) (multi-) wavelets, with (discontinuous) piecewise quartic duals as constructed in CS11. The primal wavelets satisfy Dirichlet boundary conditions of order 1 at both boundaries 0 and 1, i.e., $\vec{\sigma}=\left(\sigma_{\ell}, \sigma_{r}\right)=(1,1)$, whereas at the dual side no boundary conditions can be imposed, i.e., $\overrightarrow{\tilde{\sigma}}=\left(\tilde{\sigma}_{\ell}, \tilde{\sigma}_{r}\right)=(0,0)$.

For the present work, we generalized this construction to obtain also wavelet collections that satisfy no boundary conditions (at primal side) at either or both boundaries, i.e., $\vec{\sigma} \in\{0,1\}^{2} \backslash\{(1,1)\}$. Actually, we also slightly modified the biorthogonal collections $\left(\Psi_{(1,1),(0,0)}, \tilde{\Psi}_{(1,1),(0,0)}\right)$ from CS11] with the aim to minimize, for $\vec{\sigma} \in\{0,1\}^{2} \backslash\{(1,1)\}$, the number of $\lambda \in \nabla_{\vec{\sigma},(0,0)}$ for which either 
$\psi_{\lambda}^{(\vec{\sigma},(0,0))} \notin \Psi_{(1,1),(0,0)}$ or $\tilde{\psi}_{\lambda}^{(\vec{\sigma},(0,0))} \notin \tilde{\Psi}_{(1,1),(0,0)}$. Indeed, recall from Remark 5.3 that the extension operator has to be applied to all primal wavelets with such indices $\lambda$ (at either left or right boundary). We obtained the result that the number of such $\lambda$ on each level at left or right boundary is equal to 2. One of them corresponds to a primal wavelet that does not vanish at the boundary and therefore has to be extended to obtain a continuous extension, whereas the primal wavelet corresponding to the other only has to be extended to guarantee locality of the resulting dual wavelets by an application of Proposition 5.2 .

As an extension operator, we apply the simple reflection suited for $\frac{1}{2}<t<\frac{3}{2}$, $0<\tilde{t}<\frac{1}{2}$.

As domains, we consider the 2-dimensional slit domain $\Omega=(0,2)^{2} \backslash\{1\} \times[1,2)$, whose closure is the union of 4 squares $\tau+[0,1]^{2}\left(\tau \in \mathbb{Z}^{2}\right)$, the 3 -dimensional "thick" L-shaped domain $\Omega=(0,2)^{2} \times(0,1) \backslash[1,2)^{2} \times(0,1)$, whose closure is the union of 3 cubes $\tau+[0,1]^{3}\left(\tau \in \mathbb{Z}^{3}\right)$, and the 3-dimensional Fichera corner domain $\Omega=(0,2)^{3} \backslash[1,2)^{3}$, whose closure is the union of 7 cubes $\tau+[0,1]^{3}\left(\tau \in \mathbb{Z}^{3}\right)$. Aiming at constructing Riesz bases for $\left[H^{\tilde{t}}(\Omega), H_{0}^{t}(\Omega)\right]_{s, 2}(s \in[0,1])$, in particular, for $H_{0}^{1}(\Omega)$, we impose homogeneous Dirichlet boundary conditions of order 1 at $\partial \Omega$.

In the slit domain case, we consider tensor product wavelet bases on $(0,1)^{2}$, on $\{(1,0)\}+(0,1)^{2}$ with no boundary conditions on its left edge, and on $\{(0,1)\}+(0,1)^{2}$ and $\{(1,1)\}+(0,1)^{2}$ with no boundary conditions on their bottom edges, all with homogeneous Dirichlet boundary conditions of order 1 on the remaining edges. By applying the scale-dependent extension, first from $\{(1,0)\}+(0,1)^{2}$ to $(0,2) \times(0,1)$, and then from both top domains $\{(0,1)\}+(0,1)^{2}$ and $\{(1,1)\}+(0,1)^{2}$ over their bottom edges to $\Omega$ (see Figure [6), we end up with a piecewise tensor product basis.

In the thick L-shaped domain case, we consider tensor product wavelet bases on $(0,1)^{3}$, and on $\{(1,0,0)\}+(0,1)^{3}$ and $\{(0,1,0)\}+(0,1)^{3}$ with no boundary conditions on their interface with $(0,1)^{3}$, all with homogeneous Dirichlet boundary conditions of order 1 on the remaining faces. By applying the scale-dependent extension from $\{(1,0,0)\}+(0,1)^{3}$ to $(0,2) \times(0,1)^{2}$, and then from $\{(0,1,0)\}+(0,1)^{3}$ to $\Omega$ (see Figure [6), a piecewise tensor product basis is obtained.

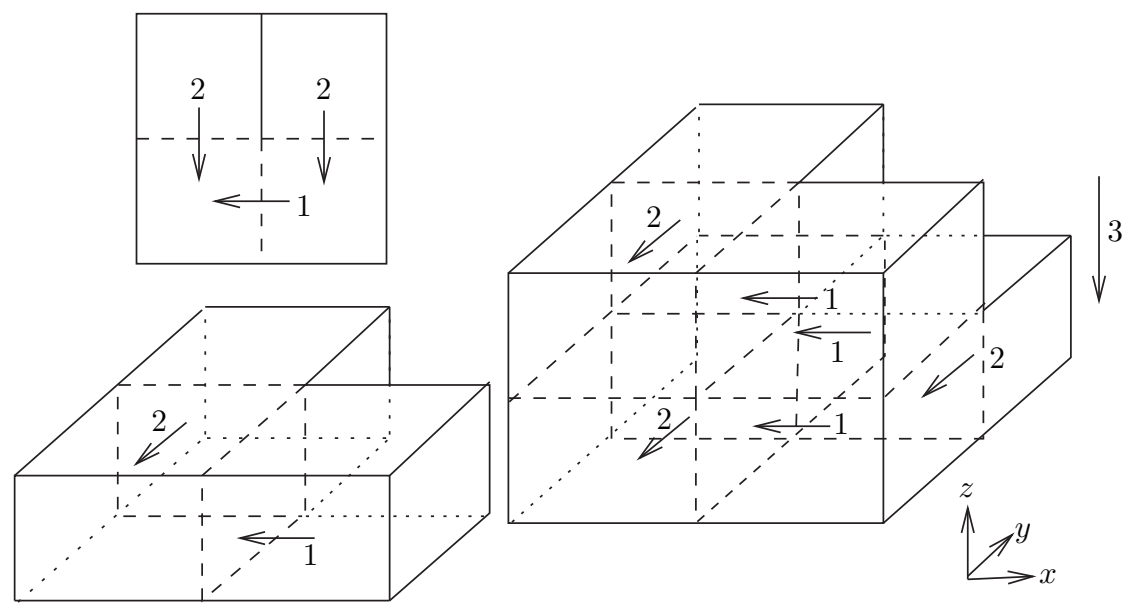

Figure 6. The direction and ordering of the extensions. 
In the Fichera corner domain case, we consider tensor product wavelet bases on $(0,1)^{3}$, on $\{(1,0,0)\}+(0,1)^{3}$ with no boundary conditions on its left face, on $\{(1,0,1)\}+(0,1)^{3}$ with no boundary conditions on its left and bottom faces, on $\{(1,1,0)\}+(0,1)^{3}$ with no boundary conditions on its left and front faces, on $\{(0,0,1)\}+(0,1)^{3}$ with no boundary conditions on its bottom face, on $\{(0,1,0)\}+$ $(0,1)^{3}$ with no boundary conditions on its front face, and on $\{(0,1,1)\}+(0,1)^{3}$ with no boundary conditions on its front and bottom faces, all with homogeneous Dirichlet boundary conditions of order 1 on the remaining faces. By applying the scale-dependent extensions in the order as indicated in Figure 6, a piecewise tensor product basis is obtained.

Using these piecewise tensor product bases, we solved the Poisson problem of finding $u \in H_{0}^{1}(\Omega)$ such that

$$
\int_{\Omega} \nabla u \cdot \nabla v=f(v) \quad\left(v \in H_{0}^{1}(\Omega)\right)
$$

by applying the adaptive wavelet-Galerkin method ([CDD01, Ste09]). This method is known to produce a sequence of approximations from the span of the basis that converges in $H^{1}(\Omega)$-norm with the best possible rate. Assuming a sufficiently smooth right-hand side, Theorem 5.6 together with the regularity results from 6.1 or 6.2 show that this rate is $d-m=5-1=4$ (indeed an even higher rate can generally not be expected).

Furthermore, if the bi-infinite stiffness matrix of the PDE w.r.t. the basis is sufficiently close to a sparse matrix, in the sense that it is $s^{*}$-compressible for some $s^{*}>4$, then this adaptive method has optimal computational complexity. The univariate wavelet basis from CS11 was designed such that any second order PDE on $(0,1)^{n}$ with homogeneous Dirichlet boundary conditions gives rise, w.r.t. the tensor product basis, to a bi-infinite stiffness matrix which is truly sparse. By losing the Dirichlet boundary conditions on one side of each interface between subdomains, and by the application of reflections, this sparsity, however, is partly lost in the sense that columns corresponding to wavelets that are non-zero at an interface contain infinitely many non-zero entries. The sizes of these entries, however, decay sufficiently fast as a function of the difference in levels of the wavelets involved so that, nevertheless, the stiffness matrix is $s^{*}$-compressible with $s^{*}=\infty$, meaning that indeed the adaptive method has optimal computational complexity.

In all three examples, to avoid approximating an infinite forcing vector, for our convenience we took as the right-hand side function $f=1$. As this right-hand side function nowhere vanishes on the boundary, it gives rise to all singular terms in the solution associated to corners and edges. Our solution method does not take advantage of symmetries in the solution due to those in the right-hand side, or of other special properties of $f=1$. As such, we expect that our results are representative for those that are obtained for any smooth right-hand side function that nowhere vanishes on the boundary.

To investigate how the application of the extensions, and the incorporation of univariate wavelet bases without boundary conditions at either or both endpoints affects the conditioning of the bi-infinite stiffness matrix, we computed numerically the condition number of the stiffness matrix ("preconditioned" by its diagonal) restricted to "full-grid" wavelet index sets. We considered the cases of the slit domain $(0,2)^{2} \backslash\{1\} \times[1,2)$ subdivided into 4 squares, the square $(-1,1)^{2}$ subdivided into 4 squares, and the square $(0,1)^{2}$ not being subdivided. The results, given in 
Table 1, show the price to be paid for the construction of a piecewise tensor product basis, as well as that seemingly a re-entrant corner does not negatively affect the condition number.

TABLE 1. Condition numbers of the diagonally preconditioned stiffness matrix restricted to the square block corresponding to row and column indices $\boldsymbol{\lambda}$ with $\||\boldsymbol{\lambda}|\|_{\infty} \leq J$. The cardinality of this set of row- or column-indices is (approximately) equal to $9.4^{J+2}$ (first two cases) and $9.4^{J+1}$ (last case), respectively.

\begin{tabular}{c|c|c|c|c|c|c|c|c|}
$J$ & 0 & 1 & 2 & 3 & 4 & 5 & 6 & 7 \\
\hline$(-1,1)^{2}$ into 4 & 790 & 1180 & 1288 & 1816 & 2335 & 2827 & 3263 & 3650 \\
slit domain into 4 & 378 & 634 & 860 & 1167 & 1509 & 1882 & 2258 & 2620 \\
\hline \hline$J$ & 1 & 2 & 3 & 4 & 5 & 6 & 7 & 8 \\
\hline \hline$(0,1)^{2}$ & 37 & 61 & 96 & 122 & 146 & 167 & 185 & 201
\end{tabular}

Let us now first consider the Poisson problem with $f=1$ on the two-dimensional slit domain. Its solution is illustrated in Figure 7

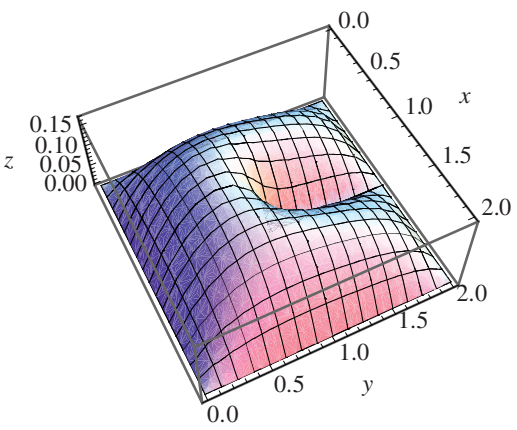

Figure 7. The solution of the Poisson problem with $f=1$ on the slit domain $(0,2)^{2} \backslash\{1\} \times[1,2)$.

In Figure 8 we give support lengths of the approximate solutions in piecewise tensor product wavelet coordinates obtained by the adaptive wavelet-Galerkin scheme vs. the (relative) $\ell_{2}$-norm of their residual in the bi-infinite matrix vector system, the latter being equivalent to the $H^{1}(\Omega)$-norm of the error. The optimal rate -4 indicated by the slope of the hypotenuse of the triangle is accurately approached for the problem sizes near the end of the computation.

At the end of this computation, the cardinality of the set of adaptively selected wavelets was approximately $1.5 \cdot 10^{5}$. The maximum of $\||\boldsymbol{\lambda}|\|_{\infty}$ or $\||\boldsymbol{\lambda}|\|_{1}$ over all $\boldsymbol{\lambda}$ from this set was equal to 39 or 78 , respectively, essentially meaning that locally, near the re-entrant corner the approximation space has the character of a "full-grid". The smallest non-adaptive "full-grid" or "sparse-grid" index set that contains all adaptively selected wavelets has cardinality equal to approximately $4.4 \cdot 10^{25}$ and $6.8 \cdot 10^{27}$, respectively, illustrating the strong local refinement.

Centers of supports of the piecewise tensor product wavelets that were selected by the adaptive wavelet-Galerkin scheme are indicated in Figure 9 


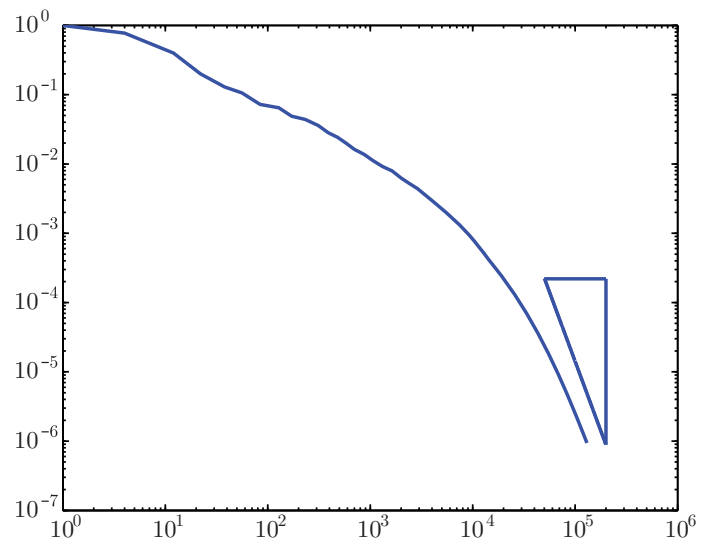

FIGURE 8. Support length vs. relative residual of the approximations produced by the adaptive wavelet-Galerkin scheme for the Poisson problem with $f=1$ on the slit domain $(0,2)^{2} \backslash\{1\} \times[1,2)$ with the piecewise tensor product basis.
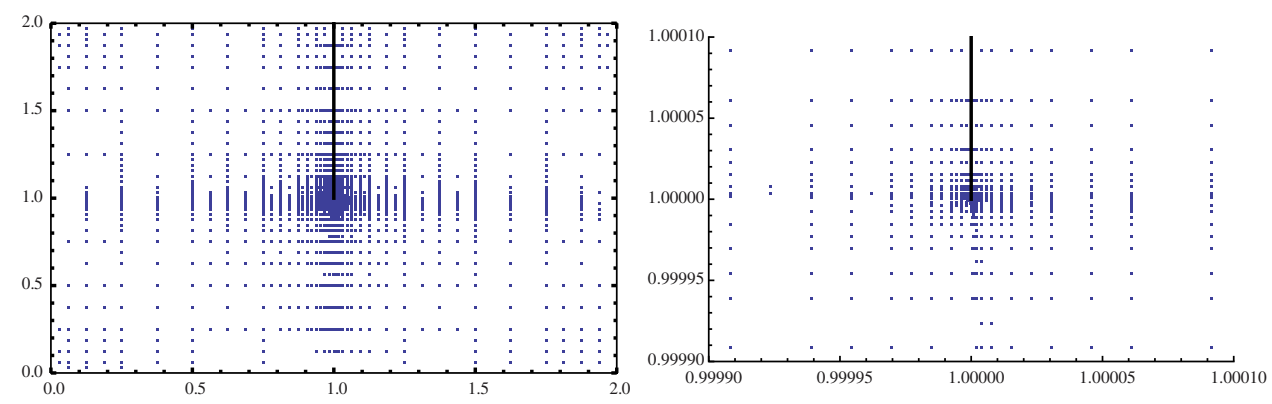

Figure 9. Centers of the supports of the piecewise tensor product wavelets that were selected by the adaptive wavelet-Galerkin scheme for the slit domain. The number of wavelets here is 25339 . The right picture is a zoom in of the left one.

Next, we give numerical results for the Poisson problem with $f=1$ on the thick L-shaped domain $\Omega=(0,2)^{2} \times(0,1) \backslash[1,2)^{2} \times(0,1)$. In Figure 10, we give the support lengths, in piecewise tensor product wavelet coordinates, of the approximate solutions obtained by the adaptive wavelet-Galerkin scheme vs. the (relative) $\ell_{2}$-norm of their residual in the bi-infinite matrix vector system, the latter being equivalent to the $H^{1}(\Omega)$-norm of the error. The optimal rate -4 indicated by the slope of the hypotenuse of the triangle is quite accurately approached for the problem's sizes near the end of the computation.

The centers of supports of the piecewise tensor product wavelets that were selected by the adaptive wavelet-Galerkin scheme are illustrated in Figure 11.

At the end of the computation, the cardinality of the set of adaptively selected wavelets was approximately $3 \cdot 10^{6}$. The maximum of $\||\boldsymbol{\lambda}|\|_{\infty}$ or $\||\boldsymbol{\lambda}|\|_{1}$ over all $\boldsymbol{\lambda}$ from this set was equal to 46 or 92 , respectively. The maximum of $\||\boldsymbol{\lambda}|\|_{1}$ was attained for $\boldsymbol{\lambda}$ with $|\boldsymbol{\lambda}|=(46,46,0)$; cf. the clustering of points around $\left(1,1, \frac{1}{2}\right)$ in 


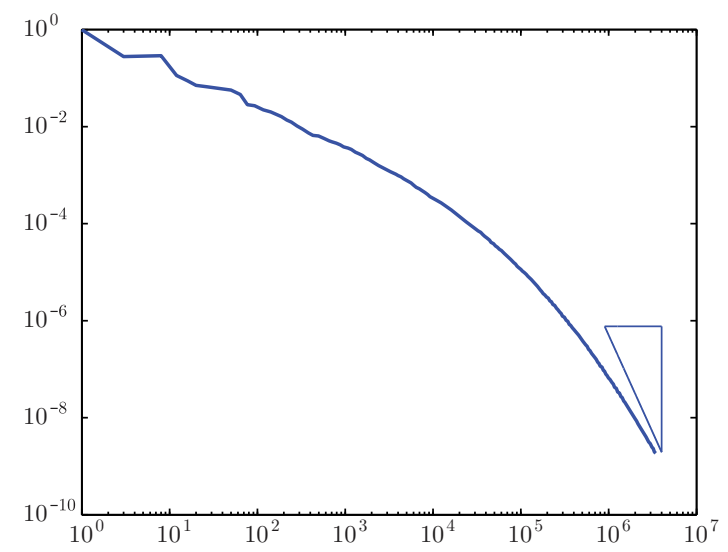

FIGURE 10. Support length vs. relative residual of the approximations produced by the adaptive wavelet-Galerkin scheme for the Poisson problem with $f=1$ on the thick L-shaped domain $\Omega=(0,2)^{2} \times(0,1) \backslash[1,2)^{2} \times(0,1)$ with the piecewise tensor product basis.

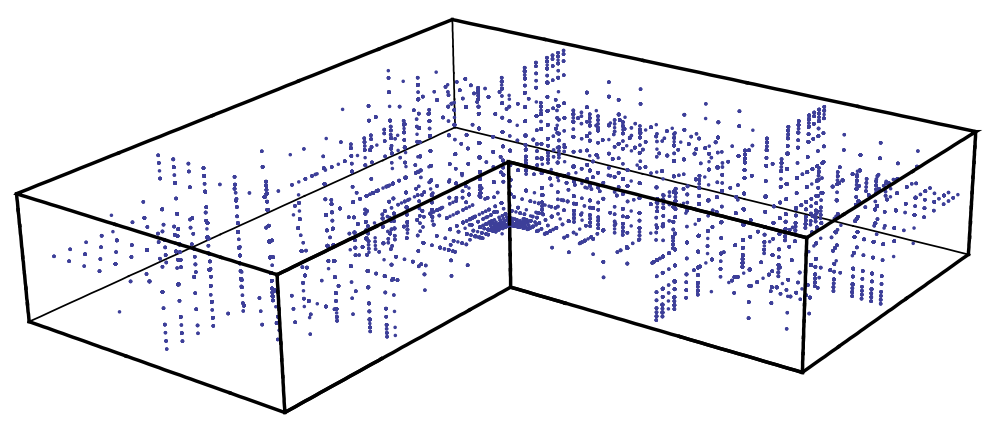

FiguRE 11. Centers of the supports of the piecewise tensor product wavelets that were selected by the adaptive wavelet-Galerkin scheme for the thick L-shaped domain. The number of wavelets here is 20421.

Figure 11. The smallest non-adaptive "full-grid" or "sparse-grid" index set that contains all adaptively selected wavelets has cardinality equal to approximately $2.3 \cdot 10^{44}$ and $2.8 \cdot 10^{34}$, respectively.

Finally, we give numerical results for the Poisson problem with $f=1$ on the Fichera corner domain $\Omega=(0,2)^{3} \backslash[1,2)^{3}$. In Figure 12, we give the support lengths, in piecewise tensor product wavelet coordinates, of the approximate solutions obtained by the adaptive wavelet-Galerkin scheme vs. the (relative) $\ell_{2}$-norm of their residual in the bi-infinite matrix vector system, the latter being equivalent to the $H^{1}(\Omega)$-norm of the error. Due to strong singularities caused by the reentrant corners and edges, even with a problem size at the end of our computation of approximately $2.5 \cdot 10^{6}$, the rate is not yet very close to the asymptotic rate -4 . 


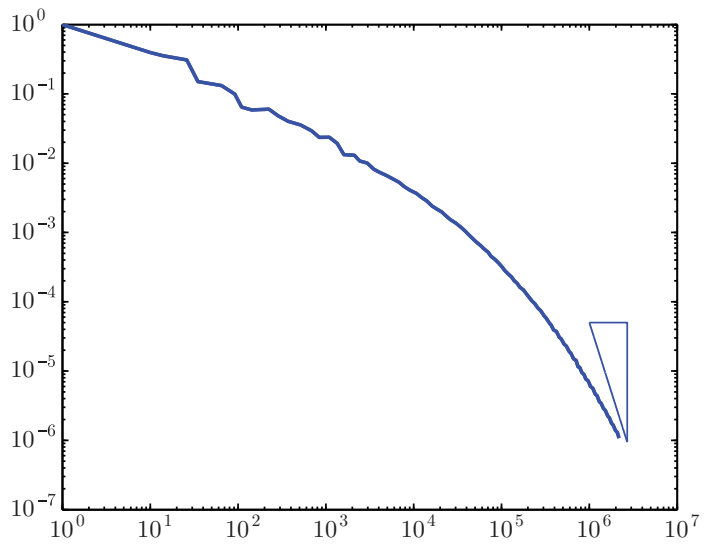

FIGURE 12. Support length vs. relative residual of the approximations produced by the adaptive wavelet-Galerkin scheme for the Poisson problem with $f=1$ on the Fichera corner domain $\Omega=(0,2)^{3} \backslash[1,2)^{3}$ with the piecewise tensor product basis.

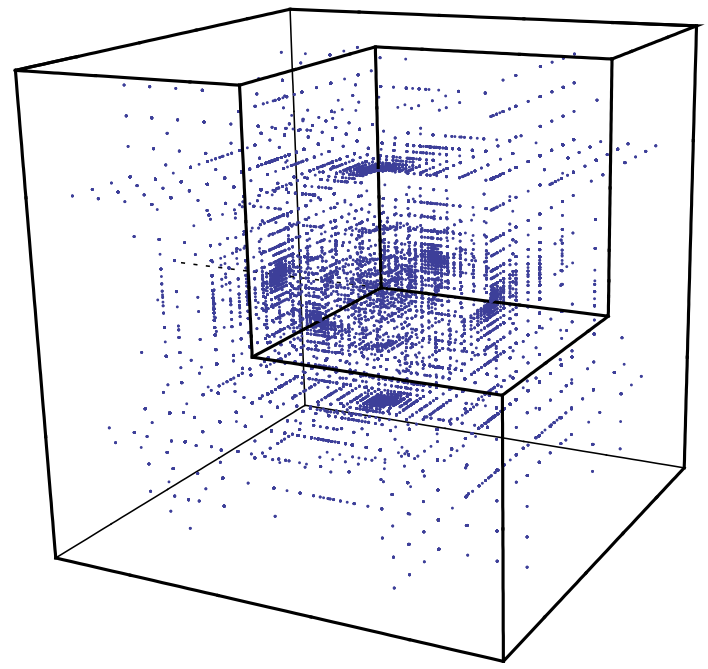

FiguRE 13. Centers of the supports of the piecewise tensor product wavelets that were selected by the adaptive wavelet-Galerkin scheme for the Fichera corner domain. The number of wavelets here is 30104 .

Nevertheless, we consider a reduction of the initial error by more than a factor $10^{6}$ to be a convincing result for this notoriously difficult problem. Recall that a rate -4 in the $H^{1}(\Omega)$-norm with an isotropic method would require approximation of order 13 , if already attainable at all in view of regularity constraints. 
The centers of supports of the piecewise tensor product wavelets that were selected by the adaptive wavelet-Galerkin scheme are illustrated in Figure 13. The maximum of $\||\boldsymbol{\lambda}|\|_{\infty}$ or $\||\boldsymbol{\lambda}|\|_{1}$ over all $\boldsymbol{\lambda}$ from the set of adaptively selected wavelets at the end of our computation was equal to 32 or 64 , respectively. The maximum of $\||\boldsymbol{\lambda}|\|_{1}$ was attained for $\boldsymbol{\lambda}$ with $|\boldsymbol{\lambda}|$ equal to $(32,32,0),(32,0,32)$ or $(0,32,32)$; cf. the clustering of points around $(1,1,1) \pm \frac{1}{2} e_{i}(1 \leq i \leq 3)$ in Figure 13 . The smallest non-adaptive "full-grid" or "sparse-grid" index set that contains all adaptively selected wavelets has cardinality approximately equal to $1.2 \cdot 10^{32}$ and $3.6 \cdot 10^{25}$, respectively.

\section{REFERENCES}

[Ape99] T. Apel. Anisotropic finite elements: Local estimates and applications. Advances in Numerical Mathematics. B. G. Teubner, Stuttgart, 1999. MR.1716824(2000k:65002)

[CDD01] A. Cohen, W. Dahmen, and R. DeVore. Adaptive wavelet methods for elliptic operator equations - Convergence rates. Math. Comp, 70:27-75, 2001. MR 1803124|(2002h:65201)

[CDN10] M. Costabel, M. Dauge, and S. Nicaise. Analytic regularity for linear elliptic systems in polygons and polyhedra. Technical report, 2010. arXiv:1002.1772v1 [math.AP].

[CF83] Z. Ciesielski and T. Figiel. Spline bases in classical function spaces on compact $C^{\infty}$ manifolds. I and II. Studia Math., 76(2):1-58 and 95-136, 1983. MR728195|(85f:46060a)

[CS11] N.G. Chegini and R.P. Stevenson. The adaptive tensor product wavelet scheme: Sparse matrices and the application to singularly perturbed problems. IMA J. Numer. Anal., 32(1):75-104, 2011.

[Dij09] T.J. Dijkema. Adaptive tensor product wavelet methods for solving PDEs. PhD thesis, Utrecht University, 2009.

[DKU99] W. Dahmen, A. Kunoth, and K. Urban. Biorthogonal spline-wavelets on the interval - Stability and moment conditions. Appl. Comp. Harm. Anal., 6:132-196, 1999. MR:1676771 (99m:42046)

[DS99] W. Dahmen and R. Schneider. Wavelets on manifolds I: Construction and domain decomposition. SIAM J. Math. Anal., 31:184-230, 1999. MR.1742299 (2000k:65242)

[DS10] M. Dauge and R.P. Stevenson. Sparse tensor product wavelet approximation of singular functions. SIAM J. Math. Anal., 42(5):2203-2228, 2010. MR2729437(2011k:42073)

[Hes41] M.R. Hestenes. Extension of the range of differentiable functions. Duke Math. J., 8(1):183-192, 1941. MR0003434 (2:219c)

[KS06] A. Kunoth and J. Sahner. Wavelets on manifolds: An optimized construction. Math. Comp., 75:1319-1349, 2006. MR2219031(2007d:42076)

[MR03] V. G. Maz'ya and J. Roßmann. Weighted $L_{p}$ estimates of solutions to boundary value problems for second order elliptic systems in polyhedral domains. ZAMM Z. Angew. Math. Mech., 83(7):435-467, 2003. MR1987897 (2004g:35061)

[Nit05] P.-A. Nitsche. Sparse approximation of singularity functions. Constr. Approx., 21(1):6381, 2005. MR2105391 (2005h:41038)

[Nit06] P.-A. Nitsche. Best $N$-term approximation spaces for tensor product wavelet bases. Constr. Approx., 24(1):49-70, 2006. MR2217525(2007a:41032)

[Pri10] M. Primbs. New stable biorthogonal spline-wavelets on the interval. Results Math., 57(1-2):121-162, 2010. MR2603016(2011e:42079)

[Ste09] R.P. Stevenson. Adaptive wavelet methods for solving operator equations: An overview. In R.A. DeVore and A. Kunoth, editors, Multiscale, Nonlinear and Adaptive Approximation: Dedicated to Wolfgang Dahmen on the Occasion of his 60th Birthday, pages 543-598. Springer, Berlin, 2009. MR2648381(2011k:65196)

[SU09] W. Sickel and T. Ullrich. Tensor products of Sobolev-Besov spaces and applications to approximation from the hyperbolic cross. J. Approx. Theory, 161:748-786, 2009. MR2563079 (2010j:46056) 
Korteweg-de Vries Institute for Mathematics, University of Amsterdam, P.O. Box 94248, 1090 GE Amsterdam, The Netherlands

E-mail address: n.godarzvandchegini@uva.nl

Department of Mathematics and Computer Sciences, Philipps-University Marburg, Hans-Meerwein Str., Lahnberge, 35032 Marburg, Germany

E-mail address: dahlke@mathematik. uni-marburg.de

Department of Mathematics and Computer Sciences, Philipps-University Marburg, Hans-Meerwein Str., Lahnberge, 35032 Marburg, Germany

E-mail address: friedrich@mathematik.uni-marburg.de

Korteweg-de Vries Institute for Mathematics, University of Amsterdam, P.O. Box 94248, 1090 GE Amsterdam, The Netherlands

E-mail address: r.p.stevenson@uva.nl 